Notice: This manuscript has been authored by UT-Battelle, LLC, under Contract No. DEAC0500OR22725 with the U.S. Department of Energy. The United States Government retains and the publisher, by accepting the article for publication, acknowledges that the United States Government retains a non-exclusive, paid-up, irrevocable, world-wide license to publish or reproduce the published form of this manuscript, or allow others to do so, for the United States Government purposes. The Department of Energy will provide public access to these results of federally sponsored research in accordance with the DOE Public Access Plan (http://energy.gov/downloads/doe-public-access-plan). 


\title{
High-resolution dielectric characterization of minerals: a step towards understanding the basic interactions between microwaves and rocks
}

\author{
T. Monti ${ }^{a^{*}}$, A. Tselev ${ }^{b}$, O. Udoudo ${ }^{a}$, I. N. Ivanov ${ }^{b}$, C. Dodds ${ }^{a}$, S. W. Kingman ${ }^{a}$ \\ ${ }^{a}$ Faculty of Engineering, University of Nottingham, NG7 2RD, Nottingham, UK \\ ${ }^{\mathrm{b}}$ Center for Nanophase Materials Sciences, Oak Ridge National Laboratories, Oak Ridge, TN 37831 , \\ USA
}

\begin{abstract}
Microwave energy was demonstrated to be potentially beneficial for reducing the cost of several steps of the mining process. Significant literature was developed about this topic but few studies are focused on understanding the interaction between microwaves and minerals at a fundamental level in order to elucidate the underlying physical processes that control the observed phenomena. This is ascribed to the complexity of such phenomena, related to chemical and physical transformations, where electrical, thermal and mechanical forces play concurrent roles. In this work a new characterization method for the dielectric properties of mineral samples at microwave frequencies is presented. The method is based upon the scanning microwave microscopy technique that enables measurement of the dielectric constant, loss factor and conductivity with extremely high spatial resolution and accuracy. As opposed to conventional dielectric techniques, the scanning microwave microscope can then access and measure the dielectric properties of micrometric-sized mineral inclusions within a complex structure of natural rock. In this work two micrometric hematite inclusions were characterized at a microwave frequency of $3 \mathrm{GHz}$. Scanning electron microscopy/energydispersive $\mathrm{x}$-ray spectroscopy and confocal micro-Raman spectroscopy were used to determine the structural details and chemical and elemental composition of mineral sample on similar scale.
\end{abstract}

Key words: Scanning microwave microscope; Dielectric properties; Raman spectra; SEM-EDX; Minerals; Hematite

*Corresponding author; email: tamara.monti@nottingham.ac.uk 


\section{Introduction}

Microwave heating was demonstrated to be potentially beneficial in reducing the cost of various mineral processing unit operations (Haque, 1999), thereby making the mining process more efficient and sustainable (Kingman, 2006). For example, the effectiveness of the microwave-induced thermally assisted liberation was extensively demonstrated (Kingman et al., 2004; Walkiewicz et al., 1989). Microwave fields induce volumetric and selective heating; in a heterogeneous material only the 'lossy' phases absorb electromagnetic energy, avoiding the waste through the 'bulk-heating' of the surrounding rock (Jones et al., 2005). Due to the different expansion rates of the minerals in the rock, micro-fractures are induced, theoretically making the grinding process less energy-consuming (Kingman and Rowson, 1998).

In spite of its potential, the application of microwave energy in the mining process has not delivered the outcomes required in order to become a commonly used technology in this field. The reasons are multiple but certainly can be ascribed to a lack of fundamental understanding of the interacting mechanisms between minerals and the microwave field itself. In particular, few pioneering studies were proposed for correlating physical effects (mechanical, thermal) to the electromagnetic energy absorbed (Jones et al., 2005; Whittles et al., 2006; Ali and Bradshaw, 2009, 2010, 2011), and a sparse literature base was developed so far for correlating these effects to the electromagnetic properties that are the most important for understanding the microwave-matter interactions.

A very first example of study of the combined effects of thermo-physical, mechanical and electromagnetic properties involved in the microwave-assisted fragmentation and comminution has been presented in the recent literature (Hartlieb, et al., 2012, 2015; Meisels, et al, 2015; Toifl, et al., 2016).

The heating mechanism of minerals in electromagnetic fields is correlated to complex physical phenomena, related to dielectric and magnetic losses:

$$
P_{a b s}=\omega \varepsilon_{0} \varepsilon_{e f f}^{\prime \prime} E_{r m s}^{2} V+\omega \mu_{0} \mu_{e f f}^{\prime \prime} H_{r m s}^{2} V
$$

where $P_{a b s}$ is the average power deposited within the sample in watts [W], $\omega=2 \mu f$ is the wave angular frequency in hertz [Hz], $\varepsilon_{0}$ and $\mu_{0}$ are the vacuum permittivity and permeability, respectively in farads per metre $[\mathrm{F} / \mathrm{m}]$ and henry per metre $[\mathrm{H} / \mathrm{m}], \varepsilon_{e f f}^{\prime \prime}$ and $\mu_{e f f}^{\prime \prime}$ are the electric and magnetic effective loss factors, $E_{r m s}$ and $H_{r m s}$ are the average intensities of the electric and magnetic field, respectively in volts per metre $[\mathrm{V} / \mathrm{m}]$ and amperes per metre $[\mathrm{A} / \mathrm{m}], V$ is the volume of the heated sample in $\left[\mathrm{m}^{3}\right]$.

The deposited power is therefore dependent upon the material characteristics which are variable with frequency (Metaxas and Meredith, 1983):

$$
\begin{gathered}
\varepsilon_{e f f}^{\prime \prime}=\varepsilon^{\prime \prime}+\sigma / \omega \varepsilon_{0}(2) \\
\mu_{e f f}^{\prime \prime}=\mu^{\prime \prime}
\end{gathered}
$$

It is then essential to evaluate the complex dielectric permittivity $\varepsilon^{*}=\varepsilon^{\prime}-j \varepsilon_{e f f}^{\prime \prime}$ and magnetic permeability $\mu^{*}=\mu^{\prime}-j \mu_{e f f}^{\prime \prime}$ of the sample under analysis. The first quantity, at microwave frequencies, is mainly determined by the polarization effect, quantified by $\varepsilon_{e f f}^{\prime \prime}$. The only exception is for highly conducting materials where the conduction phenomenon, quantified by $\sigma$, is dominant. $\mu_{e f f}^{\prime \prime}$ is usually negligible for non-magnetic samples.

The heterogeneity of ores makes the interaction with the microwave field highly complex. Selective heating of certain mineral inclusions with respect to the surrounding matrix typically occurs (Kingman et al., 2000; Walkiewicz et al., 1988). This is because such mineral inclusions have significantly higher dielectric properties than the rest of the rock forming material, namely a higher $\varepsilon^{\prime \prime}$. From Eq. (1), the power absorbed by the mineral inclusions is therefore higher than the surrounding gangue material, so the microwave energy 'focuses' selectively on such inclusions, giving rise to a temperature differential which is the basis of many applications of microwave energy in mineral processing.

The dielectric properties of each phase are required to describe such a complicated selective heating phenomenon from an electromagnetic point of view and then potentially relate it to a multi-physics description of the process. This need of a comprehensive description of the multi-phase constitution 
of rocks was highlighted by Meisels, et al. (2015). Unfortunately, conventional methods for the dielectric characterization of materials are not suited to this as they allow only the quantification of the properties of the material at centimetre scale.

Different techniques were applied for mineral characterization in the Ultra High Frequency (UHF) microwave range $(300 \mathrm{MHz}-3 \mathrm{GHz}$ ), where the Industrial-Scientific-Medical (ISM) spectra are located. For example, the short circuited waveguide method, based on the previously proposed standing wave perturbation method by Roberts and Von Hippel (1946), was used for the analysis of minerals between 1 and $22 \mathrm{GHz}$ (Nelson et al., 1989). In Tinga (1989), a bridge configuration, with samples mounted in the central section of a waveguide, was used for testing the dielectric properties of some metal oxides at $2.45 \mathrm{GHz}$ over a wide range of temperatures. An open-ended coaxial line technique was widely exploited as well: in reference Salsman and Holderfield (1994) chalcopyrite, chalcocite and cobaltite were analysed across a limited temperature range $\left[20-300^{\circ} \mathrm{C}\right]$. The cavity perturbation method was used extensively for even higher temperature range $\left[20-800^{\circ} \mathrm{C}\right]$ (Pickles, et al., 2005).

The main limitation of these techniques is that they cannot quantify the properties of the individual minerals in rocks. Additionally, the majority of conventional dielectric measurement techniques are highly dependent on the density of the sample under study (Nelson et al., 1989). Application of complex mixture theories is then needed for calculating the dielectric properties of single minerals from a conventional dielectric measurement (Shivola, 1999). These require additional information about particle size, shape and distribution and they represent just a simplified approximation of the real scenario. In this way, 'controllable' synthetic compounds can be measured accurately (Salsman, et al., 1996).

In this paper a novel method for measuring the dielectric properties of mineral inclusions embedded in a natural rock at microwave frequencies is presented. Such a method is based upon Scanning Microwave Microscopy (SMM) that is able to achieve extremely high spatial resolution and accurately measure the dielectric constant and losses of the sample under analysis.

The SMM technique is introduced and exemplified for the first time to hematite inclusions embedded in a gangue matrix. A comprehensive chemical and dielectric characterization of such micrometric inclusions is shown. The SMM capability of mapping the dielectric properties with sub-micrometric resolution is highlighted.

\section{Materials and methods}

A set of ore samples were core drilled to produce sample mounts of $25 \mathrm{~mm}$ diameter and $15 \mathrm{~mm}$ height. These samples were from the batch of rocks used for a microwave heating test work, which results were recently reported by John, et al. (2015). The heated phase selected for that investigation was a copper concentrate sample. All the rocks originate from a North American mine.

The mounts were cleaned with distilled water and dried using compressed air. The cleaned mounts were polished using a Struers Rotopol polishing machine to a surface roughness of $10 \mathrm{~nm}$, necessary for achieving the finish required by both the scanning microwave and Raman microscopes.

For Raman spectra comparison, powders of pure minerals were prepared. The magnetite was sourced from the Sydvaranger open pit Magnetite Mine in Northern Norway. The pyrite was sourced from the Inuvik quarry, Northwest Territories of Canada purchased from David K. Joyce Minerals.

In Fig. 1, adjacent images of a portion of the sample surface are shown. In Fig. 1a., a $5 \mathrm{X}$ magnification is used for the 'montage' map of a $6 \times 5 \mathrm{~mm}$ area; a 100X magnification is used for the Fig. 1 b. ( $0.35 \times 0.3 \mathrm{~mm}$ area). Fig. 1 gives a visual indication of the complex topographical scenario and related complex chemical and elemental composition of the sample under analysis.

The different grey tones are ascribable to the different materials that compose the rock. It is possible to identify a large number of phases in both of the images, with random shapes and positions. This means that the phases have different sizes and that they are effectively mixed to give a complex heterogeneous compound.

As it is necessary to chemically characterize this compound at a single-phase level in order to have a comprehensive description of the composition, it is therefore required to have an instrument that characterizes the dielectric properties at similar level. 
No further elements extraneous to the selected rocks were added during sample preparation in order to exclude any possible uncertainty due to contamination.
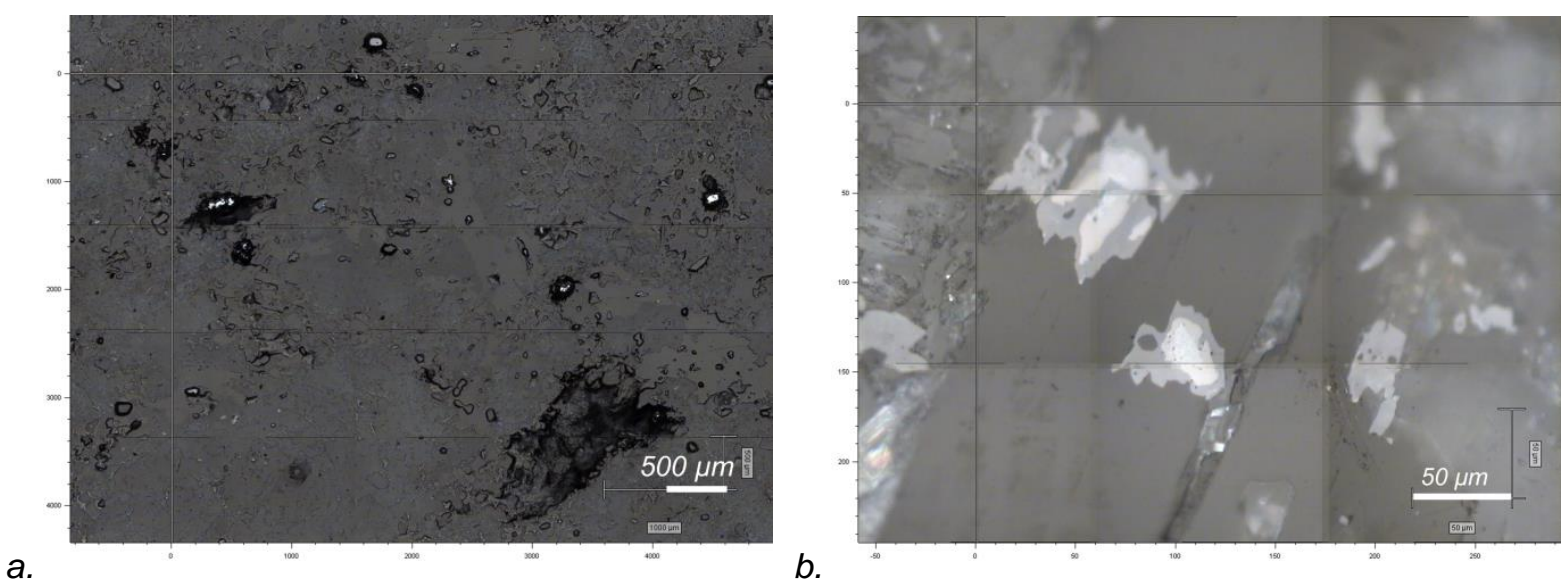

Fig. 1 Optical images of a natural rock sample surface. The images are composed of several sub images stitched to form the final one ('montage' map). Fig. 1a. shows a $6 \times 5 \mathrm{~mm}$ area (5X magnification) and Fig. $1 \mathrm{~b}$ shows a $100 \mathrm{X}$ magnification of a $0.35 \times 0.3 \mathrm{~mm}$ area. A high heterogeneity of the surface is visible at different scales. Different grey tones are ascribable to different materials. Phases are clearly recognizable at very different scales.

\subsection{Micro-Raman Spectroscopy}

In this work, the micro-Raman spectroscopy technique (Colthup, et al., 1975) is used for obtaining an elemental characterization of the sample at sub-micrometric scale. Since the spatial resolution is determined by the width of the spot of the probing light, it is possible to get a resolution that is close to that of the SMM (Collins, et al., 2014). It is then possible to detect nanometric impurities that can significantly influence the dielectric properties of the sample under investigation.

Raman spectra are collected in backscattering geometry using a Renishaw 1000 Raman confocal microscope through a 50 objective (Leica, NA $=0.75$ ) using a $532 \mathrm{~nm}$ laser as the excitation source. The Raman spectra were collected in the 100 to $1000 \mathrm{~cm}^{-1}$ frequency range by scanning the area of interest in $0.6 \mu \mathrm{m}$ steps point by point. Raman spectra of pure minerals in powder form were acquired on the same Raman spectrometer to assign inclusions to a particular mineral and to enable deconvolution of Raman spectra. Results for the minerals analysed in this paper are extensively reported in Sec. 4.

\subsection{SEM-EDX Elemental Analysis}

Micro-Raman spectroscopy and SEM-EDX techniques are complementary and allow accurate elemental and chemical characterization of the sample constituents since they are based on different probing mechanisms. They can both determine the chemical composition with micrometric and submicrometric resolution that can be directly compared to the SMM dielectric maps.

Scanning Electron Microscopy (SEM) - Energy Dispersive X-ray (EDX) spectroscopy characterization was undertaken using an FEI Quanta 600i SEM equipped with 2 Bruker XFlash 5030 detectors. The EDX information was obtained using the Bruker Espirit version 1.9 software designed by Bruker Nano $\mathrm{GMBH}$. The software was equipped to produce elemental information in the form of mineral maps (maps of the various elements identified in the sample via x-ray microanalysis) and elemental spectra from which the mineralogical composition was determined. The mineral maps are presented later.

The SEM provides backscatter (BSE) images of samples from which different mineral phase can be delineated. This information is presented in the form of grey scale images with brightness intensity values ranging from 0 to 255 , where 0 represents black and 255 white. For the ore the BSE brightness for the copper sulphide and iron oxide minerals are over 150 and appear as bright features against the much darker gangue (see an example in Fig. 2). With these feature differences, the phase 
of interest was segmented and x-rays collected from this phase where used to characterize them. BSE images and EDX analyses of the sample under investigation are presented in Sec. 4.

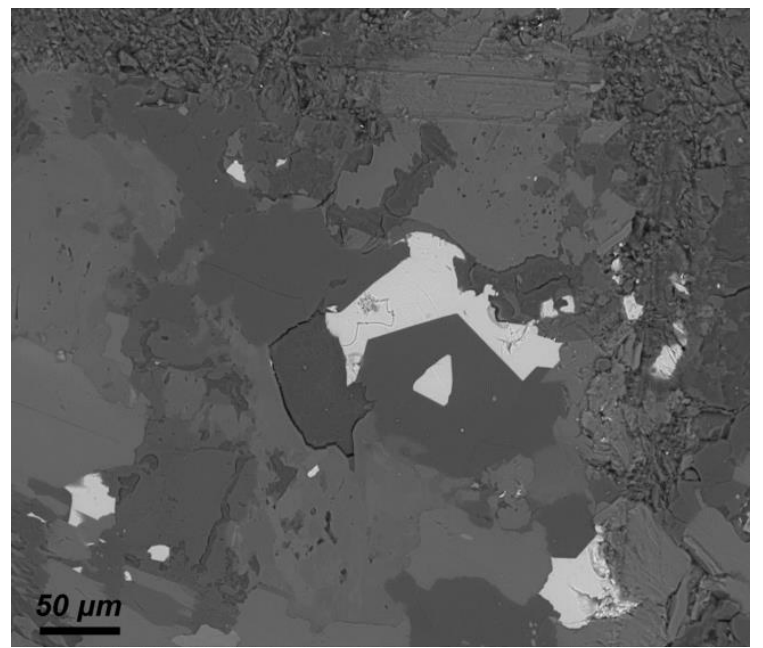

Fig. 2 An example of BSE image showing brightness intensities of different minerals. The central inclusion appears significantly brighter than the darker surrounding minerals. This is a typical feature of copper sulphide and iron oxide minerals.

\subsection{Scanning Microwave Microscopy (SMM)}

SMM is one among the family of Scanning Probe Microscopy (SPM) techniques, where a sharp probe records a change in a certain physical quantity due to interaction with a sample. This class of techniques was intensively developed since the invention of a Scanning Tunnelling Microscope (STM) in 1982 (Binnig and Rohrer, 1983) and became exponentially important because of its suitability for nanometre scale analysis. One of the best known SPM techniques is the Atomic Force Microscopy (AFM) (Binnig et al., 1986). The main feature of SPMs is an extremely high resolution that can be achieved while quantitatively measuring certain physical properties. SPM techniques are able to record local physical properties down to the nanometre length scales and their changes for each point of the scan. Through proper transducers and electronics, it is possible to obtain images of the scanned samples related to the physical interactions of interest.

The SMM records the variation of the microwave field emitted and reflected by a sharp tip placed either in close proximity or in contact with the sample. Due to the rapid decay of the interacting field and the extreme proximity between tip and sample, the range of interaction is within the so called 'reactive near-field' region of the emission. It is then possible to overcome the conventional resolution limits associated with far-field imaging and to obtain a sub-wavelength resolution (Anlage et al., 2007).

In this work, a commercial SMM system from Prime Nano Inc. ${ }^{\odot}$ (USA, http://www.primenanoinc.com/), installed on an Asylum Research (USA) MFP-3D AFM platform, was used.

As described in the scheme in Fig. 3, a microwave reflectometer, tuned at a working frequency $f=3 \mathrm{GHz}$, records in two separate channels the variations in the reactive (sMIM-C) and resistive (sMIM-R) parts of the tip-sample admittance (Fig. 4). The signal change in the sMIM-R channel is determined by the sample conductivity and increases when such conductivity (loss) increases. In turn, the signal change in the SMIM-C channel is determined by the real part of the local sample permittivity $\varepsilon^{\prime}$ and increases when $\varepsilon^{\prime}$ increases.

The sMIM system employs specialized cantilever AFM probes with microwave transmission line structures lithographically fabricated on the cantilevers. The transmission line structure on a probe runs from the probe chip through the whole cantilever length up to the sensing tip made of a Ti/W alloy. A specialized probe holder ensures interfacing of the transmission line on the probe with a coaxial cable connecting the probe with an impedance (Z-)match circuit. The typical apex radius of the tip is $50 \pm 10 \mathrm{~nm}$. In order to separate the two measurement channels and correspondingly, the two physical contributions, an initial calibration is needed on a calibration grating made of metallic patches 
on a $\mathrm{SiO}_{2} / \mathrm{Si}$ substrate. Topographical AFM images of the samples are obtained simultaneously with the SMM characterization.

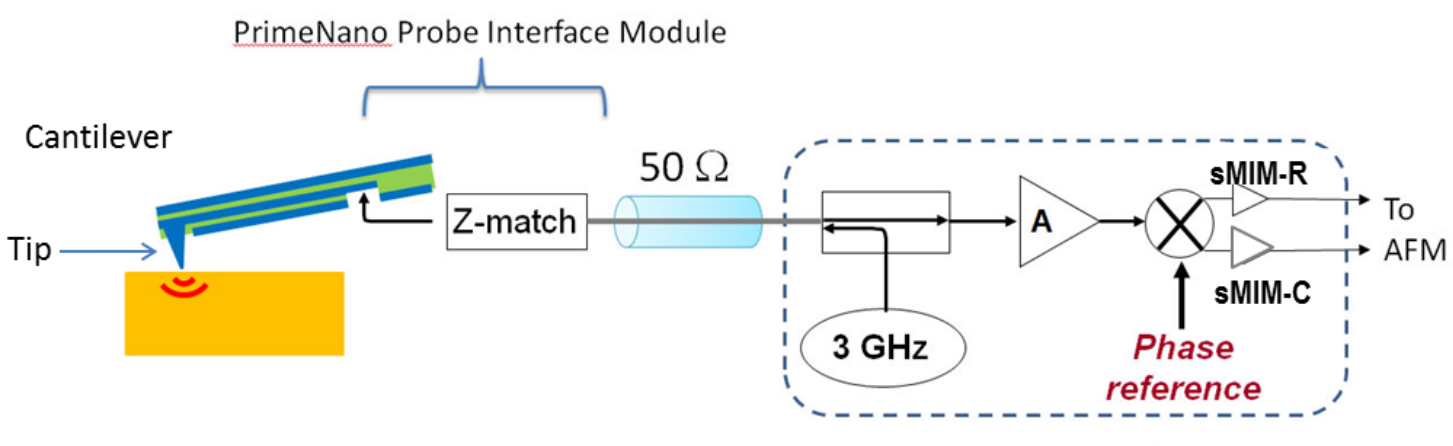

PrimeNano ScanWave Electronics

Fig. 3 Schematic of the SMM system by Prime Nano Inc.(O. A sample (yellow) is scanned under the probe that is connected to a single-frequency microwave source and a detection circuit (reflectometer). Microwaves are sent to and reflected back from the sample through a $50 \Omega$ coaxial cable, a 'z-match' circuit, and a transmission-line structure on the tip cantilever. The reflectometer electronics measures the reflection coefficient from the tip-sample system and separates the sMIM- $R$ and SMIM-C signals.

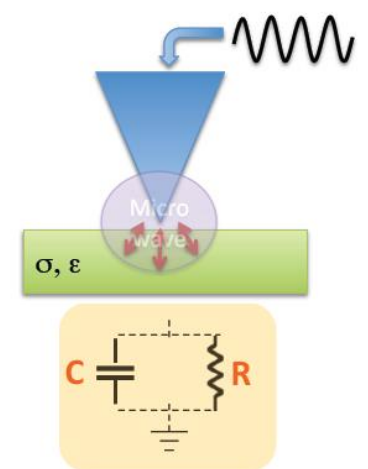

Fig. 4 Lumped elements equivalent circuit description of the SMM tip-sample interaction mechanism: the reactive interaction (modelled by a parallel capacitance) depends on the $\varepsilon^{\prime}$ of the sample, while the resistive interaction (modelled by a parallel resistance) depends on $\sigma$ (courtesy of Prime Nano Inc.(C).

In order to obtain quantitative data from the scanned samples, another calibration procedure is needed. In particular, with this latter calibration it is possible to take into account the change in the tip apex shape during the measurements. It is particularly important in this work since the tip is in contact with the sample surface while scanning, and therefore, it is subject to a small, but continuous, alteration. Therefore, the latter calibration was performed before and after each set of scans, while the former calibration on the grating was needed only when a probe was replaced with another.

Five different standard calibration samples, with known dielectric permittivity, were chosen for the latter calibration procedure: fused quartz $\mathrm{SiO}_{2}\left(\varepsilon^{\prime} \approx 4\right)$, single-crystalline (100) magnesium oxide MgO $\left(\varepsilon^{\prime} \approx 10\right)$, (100) lanthanum aluminate $\mathrm{LaAlO}_{3}\left(\varepsilon^{\prime} \approx 24\right)$, (110) titanium dioxide $\mathrm{TiO}_{2}\left(\varepsilon^{\prime} \approx 100\right)$, and $(100)$ strontium titanate $\mathrm{SrTiO}_{3}\left(\varepsilon^{\prime} \approx 300\right)$. Dielectric permittivities of the single-crystalline samples were obtained from the manufacturer's datasheets (CrysTec, GmbH, Germany). Each standard sample was a few hundred microns thick. The absolute values of the sMIM signals are subject to small drifts, which can, however, significantly affect the measurement results. Therefore, all measurements are performed in 'differential' mode with use of the so-called 'NAP mode' of the AFM microscope (Asylum Research MFP-3D). In this way, numerical values from the SMIM channels are always referenced to the "in air" condition with the tip lifted $5 \mu \mathrm{m}$ away from the sample surface. During measurements, each row of the scan is repeated twice: once with the tip in contact with the sample and once with the 
tip raised above the sample. The values of $\Delta R$ and $\Delta C$ below refer to these differences between "contact" and "in-air" conditions for both microscope channels.

By measuring the sMIM-C values related to the "in air" reference for the standards and then fitting them, it is possible to obtain an empirical calibration curve for the C-channel. Being mainly related to the reactive interaction of the microwave field with a sample, these values are influenced only by the real part of the sample dielectric permittivity $\left(\varepsilon^{\prime}\right)$. An example of such a calibration curve is shown in Fig. 5.

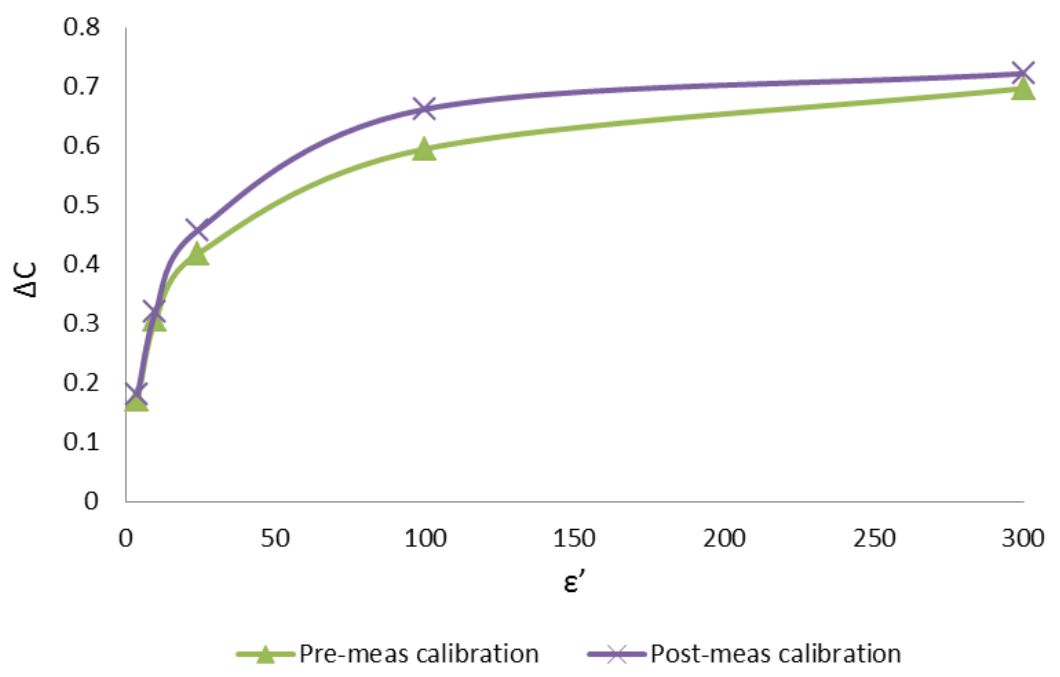

Fig. 5 Example of a calibration curve for $\varepsilon^{\prime}$ data, interpolating the values recorded by the $C$-channel of the microscope referred to the 'in air' condition $(\Delta C)$ on dielectric known samples $\left(\mathrm{SiO}_{2}\left(\varepsilon^{\prime} \approx 4\right), \mathrm{MgO}\right.$ $\left.\left(\varepsilon^{\prime} \approx 10\right), \mathrm{LaAlO}_{3}\left(\varepsilon^{\prime} \approx 24\right), \mathrm{TiO}_{2}\left(\varepsilon^{\prime} \approx 100\right), \mathrm{SrTiO}_{3}\left(\varepsilon^{\prime} \approx 300\right)\right)$. The green curve was measured before the rock analysis, the purple one was obtained afterwards. The discrepancy between the two curves reflects the changes in size and shape of the SMM probe due to the contact with the sample during the scanning process.

\section{Semi-empirical model of the SMM interaction}

In order to obtain quantitative measurements for the resistive R-channel which is influenced by the loss tangent tan $\delta$ of a sample, it is not straightforward to create a calibration curve through measuring 'known loads'. Natural materials are obviously not well-characterized in terms of loss tangent since this property is influenced by a number of factors. Additionally, it is impossible to obtain well-defined calibration standards with proper combinations of permittivity and loss tangent, especially around the values of the naturally occurring mineral phases within an ore. In this work, a semi-empirical calibration method was developed for estimating the loss tangent of samples. Namely, the tip-sample interaction was modelled by using a quasi-static numerical model in an AC/DC module of a finiteelement analysis (FEA) package COMSOL Multiphysics ${ }^{\odot}$. The reference AC voltage of amplitude $V_{a c}=1$ and a frequency $f=3 \mathrm{GHz}$ is applied to the tip in the model against a ground electrode simulating the sMIM probe shield. Fig. 6 shows the geometry of the model, where ground boundary conditions are applied to the AFM cantilever as indicated by the red arrow. 

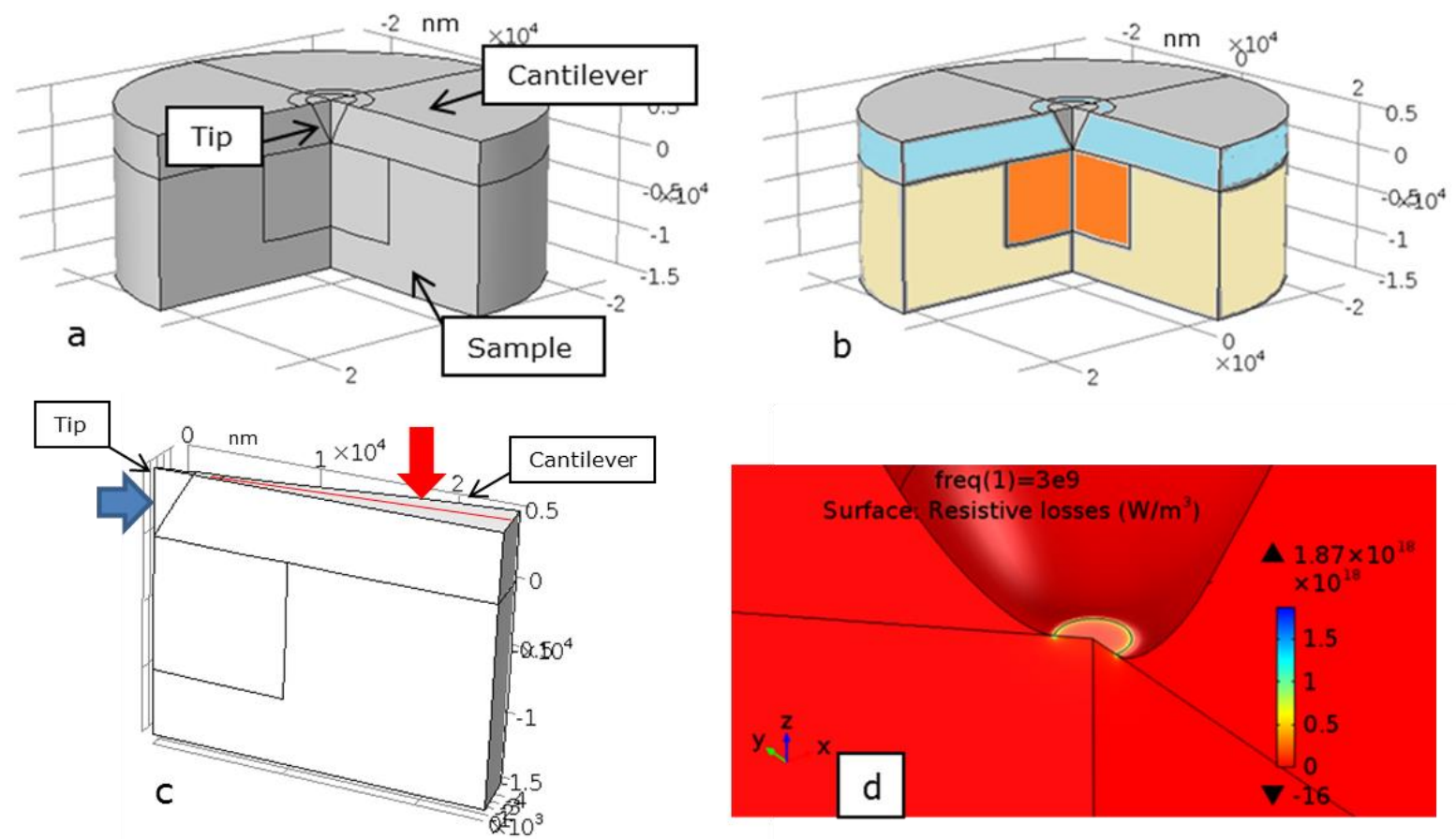

Fig. 6 a. Simplified geometry of the system implemented in COMSOL Multiphysics ${ }^{\odot}$. The actual finiteelements model is $2 D$ axisymmetric. The $3 D$ representation is obtained by rotating the section in $c$. $b$. The grey represents the metallic parts of the tip and cantilever. The cyan represents air gaps between the sample surface and the cantilever. The orange represents an inclusion of a certain material different from the surrounding (cream). c. The red arrow and line indicated where the ground condition is applied, while the reference voltage $V_{0}$ is applied to the tip (blue arrow). $d$. A magnified view of the geometrical contact between tip-sample that well represents the real physical interaction. The simplified shape of the cantilever, circular instead of rectangular, is due to the rotation operation. Anyway this does not significantly affect the results of the model in respect to the tip-sample interaction.

A quasi-static approximation is applicable since the dimensions of the model-a few micrometresare small compared to the wavelength of the electromagnetic radiation in vacuum at $3 \mathrm{GHz}$, which is $10 \mathrm{~cm}$. In the simulation, first, the permittivity of the sample is set to the same as the measured one (based on the calibration curves), and the tip-sample contact area is adjusted to reproduce the measured change in the tip-sample capacitance for 'in air' and 'in contact' conditions. After that, the conductivity of the sample is varied, and the model is solved for the complex impedance $Y$ of the tipsample system as a function of the sample conductivity $\sigma$. The ratio of the real and imaginary parts of the calculated admittance $\operatorname{Im}(Y) / \operatorname{Re}(Y)$, which corresponds to the measured ratio of the sMIM signals $\Delta R / \Delta C$ (where $\Delta R$ is the signal recorded by the R-channel of the microscope and the $\Delta C$ is recorded by the $\mathrm{C}$-channel), yields the value of the sample conductivity $\sigma$.

The obtained value of $\sigma$ is further used to obtain the sample loss tangent with the expression:

where $\varepsilon_{0}$ is vacuum permittivity.

$$
\tan \delta=\frac{\sigma}{2 \pi f \varepsilon_{0} \varepsilon^{\prime}}=\frac{\varepsilon^{\prime \prime}}{\varepsilon^{\prime}}
$$

The admittance is a function of a number of factors that are directly derived from the sMIM measurements such as the size of the objects of interest and their dielectric constant. As a starting value of the expected conductivity of the samples, loss tangent and conductivity values found in the literature (Nelson et al., 1989; Peng et al., 2012; Hotta et al., 2010) were used, after the samples were characterized by Raman and SEM-EDX techniques in terms of chemical and elemental composition. Since the tip-sample system can be considered as a lossy capacitor, with the main contributions to 
the capacitance and loss variations stemming from the sample portion right beneath the tip-sample contact, for semi-empirical estimations the following relationship can be applied:

$$
\tan \delta=\kappa \frac{\Delta R}{\Delta C},
$$

where the factor $k$ is insignificantly dependent on sample permittivity for large enough samples of size about three times the tip pyramid height of ca. $5 \mu \mathrm{m}$. This fact can be used to generate loss tangent maps based on permittivity maps using a small set of the simulations, which effectively "calibrate" the factor $\kappa$.

\subsection{Errors and uncertainties discussion}

The SMM measurements errors and uncertainties are mainly due to alteration of the SMM probe during the scans. This is because the probe tip is in contact with the sample while performing a raster scan on the sample surface. Initially in an experiment, once the roughness of the surface was ensured to be manageable by the AFM scanning and feedback systems, the microwave detection circuit was calibrated with a first calibration grating (to define sMIM-C and -R channels). After that, in order to obtain quantitative measurements of the dielectric properties, a second calibration procedure on known dielectric samples was performed as described in Sec. 2.3. The calibration curves obtained by such a procedure are influenced by the shape of the probe tip apex. This can be seen by comparing the curves in Figs. 5 and 17. The first corresponds to a sharper tip and the second to a blunter one. After a certain number of scans (even after just one scan in a case of a hard surface), the tip apex changes and so do the calibration curves associated with the probe. Evidently, uncertainties associated with the tip apex shape depend on the tip sharpness, surface conditions, tip-sample contact force, surface hardness, and scan duration. Thus, they have to be determined in each experiment with measurements of the dielectric properties. In this study, we performed calibrations before and after each image, which allowed us to keep track of the uncertainties of the measurements due to the probe tip changes, and such uncertainties can be determined by comparing 'before' and 'after' calibration curves at the obtained values of permittivity. In the semi-empirical model used for quantifying the microwave losses of the samples, the main source of uncertainties is the difference of the tip apex shapes in the reality and in the numerical model. These uncertainties can be estimated using the same numerical model and verified with a set of samples with known dielectric permittivity and loss tangent. In this work, the loss measurement results were verified against literature data on similar minerals, and were found to be within the range reported in literature already after the first adjustment of the tip-sample contact size in the model to match the sMIM-C signal, calculated capacitance, and the sample permittivity.

\section{Results}

\subsection{SEM-EDX analysis and confocal micro-Raman spectroscopy results}

The set of techniques described so far were applied for a comprehensive nanoscale characterization of natural rock constituents. In particular, the combined analysis through micro-Raman and SEM-EDX techniques, allowed for recognizing the chemical composition of the samples under analysis. The nature of the mineral inclusions was determined with nanometric resolution, as detailed in the following pictures. Two hematite inclusions within a complex matrix were tested as exemplar samples for the SMM technique.

Figs. 7, 8 and 9 show the results of the SEM-EDX analysis performed on the minerals under investigation. Polychromatic maps resulting from the EDX analysis highlight the different chemical composition of the sample under investigation. In Fig. 7, the two hematite inclusions are identified by the pink tone; the surrounding matrix is essentially composed by calcium and carbon. The brown colour comes from the combination of the $\mathrm{C}$ (cyan) and $\mathrm{Ca}$ (dark orange) contributions. Finally, the surrounding is composed by silicon and oxygen, with diffused spots of magnesium (green). Fig. 8 and 9 are the results of the SEM-EDX analyses at higher resolution around the top-left hematite inclusion. 

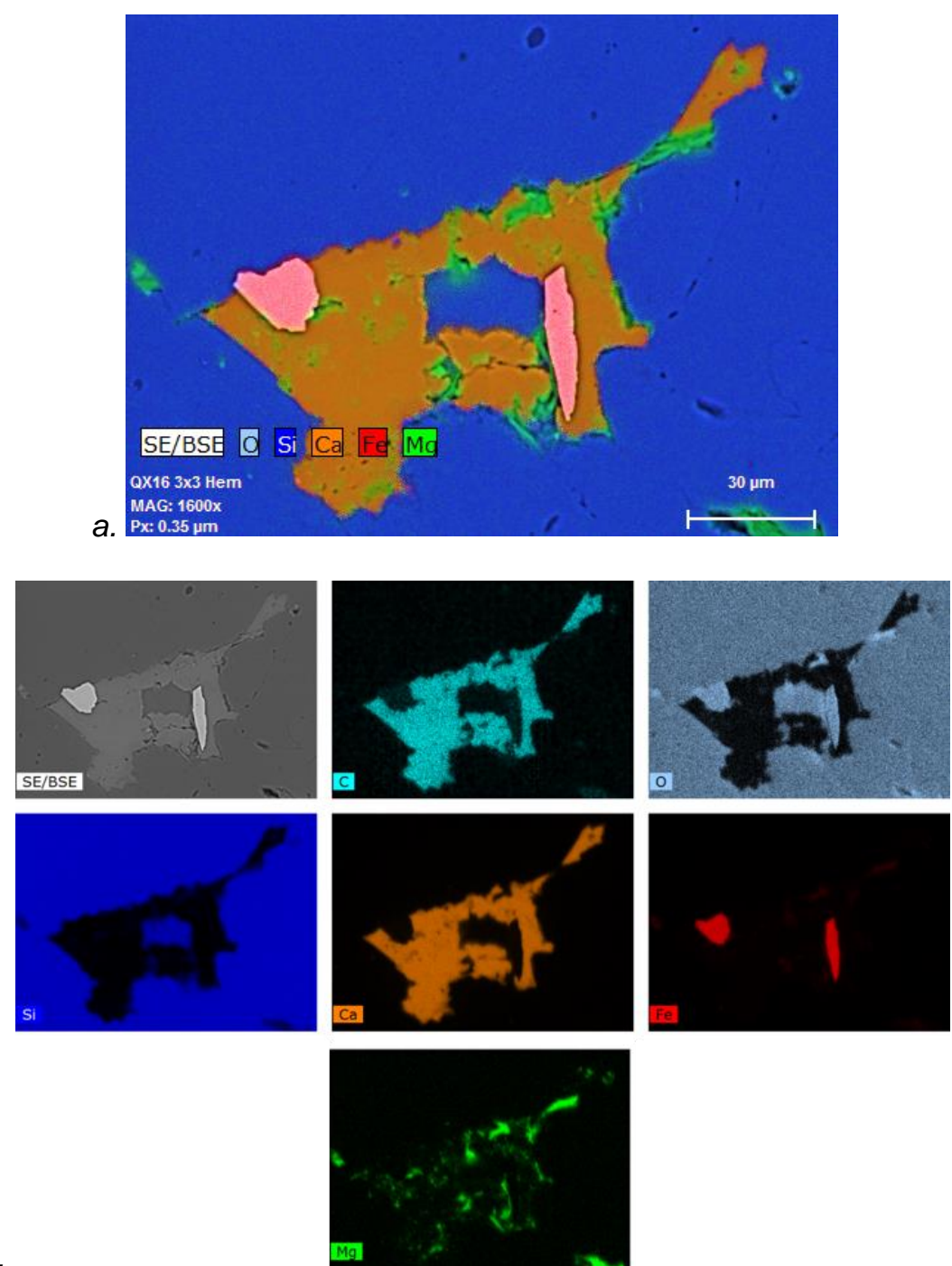

b.

Fig. 7 SEM-EDX analysis of two hematite inclusions and their surrounding dielectric gangue matrix: a. collects the contributions coming from the different chemical elements by overlapping the different colours used for them, individually reported in b. The 'pink' hematite is then the combination of the 'red' iron and the 'white/grey' oxygen. The two inclusions are just a few tens of micrometres. They are surrounded by a larger phase composed of 'brown' calcium carbonate (calcite), a combination of 'dark orange' calcium and 'cyan' carbon. This contains Mg impurities. Everything is surrounded by 'light blue' quartz ('blue' silicon + 'white/grey' oxygen).
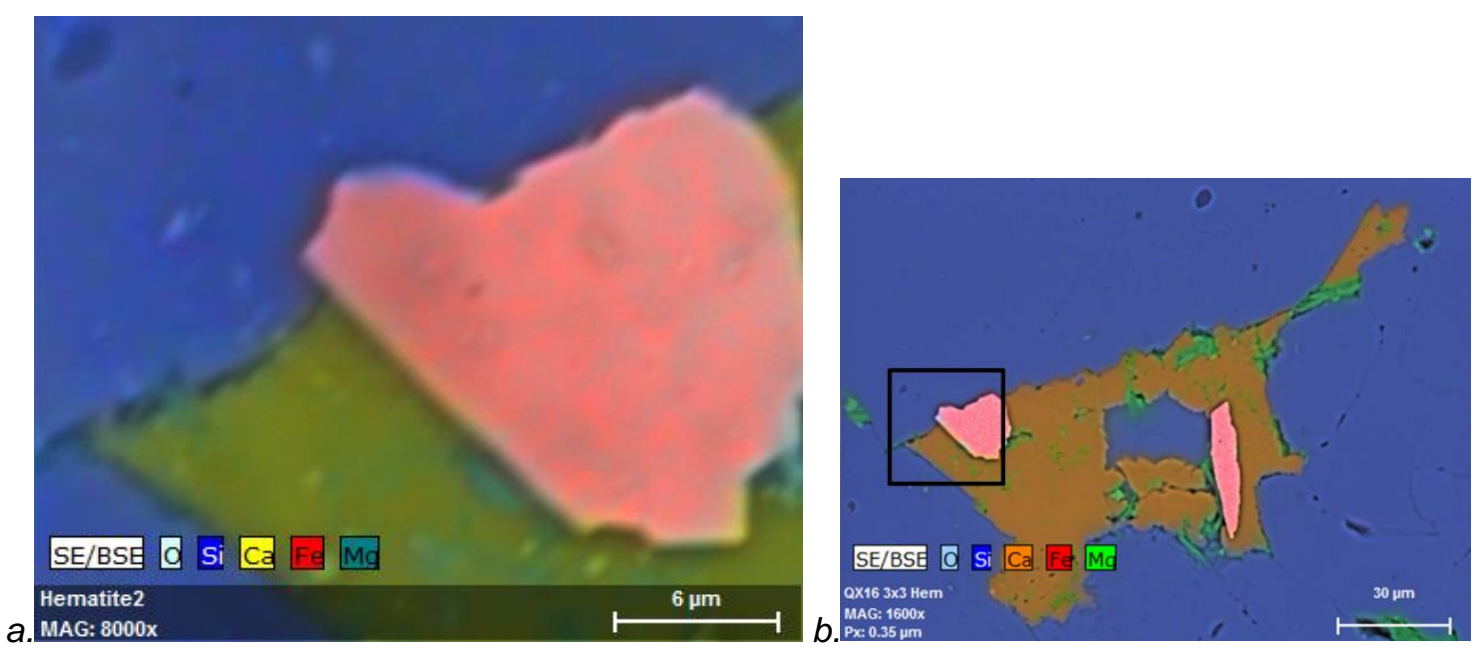

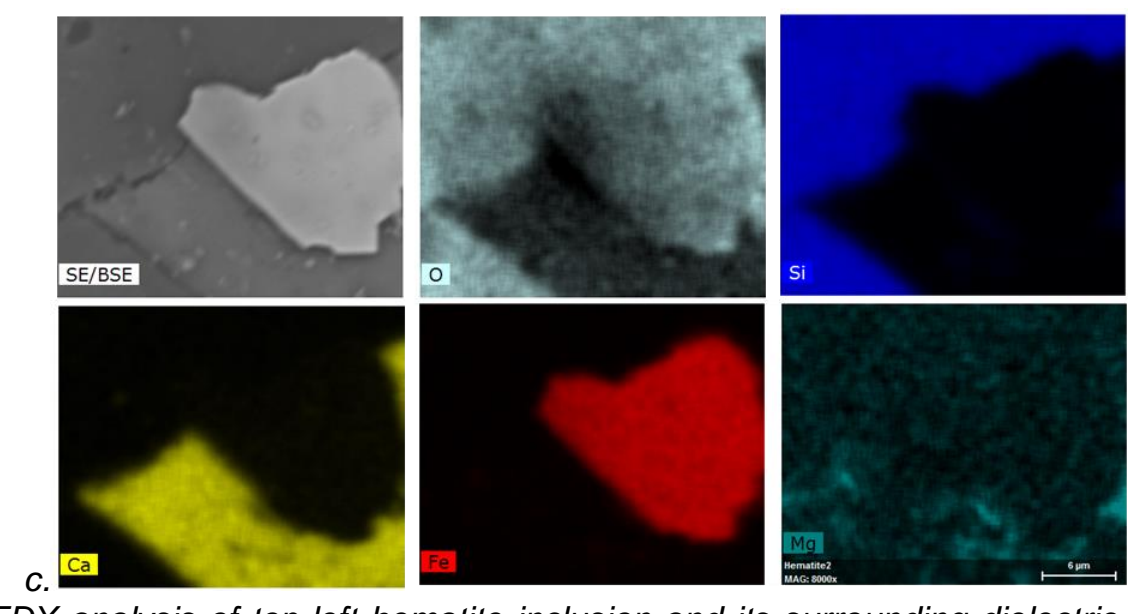

Fig. 8 SEM-EDX analysis of top-left hematite inclusion and its surrounding dielectric gangue matrix. The exact location across the large sample area is highlighted by the black square in Fig. $8 b$. a. collects the contribution from the different chemical elements, detailed in $c$.

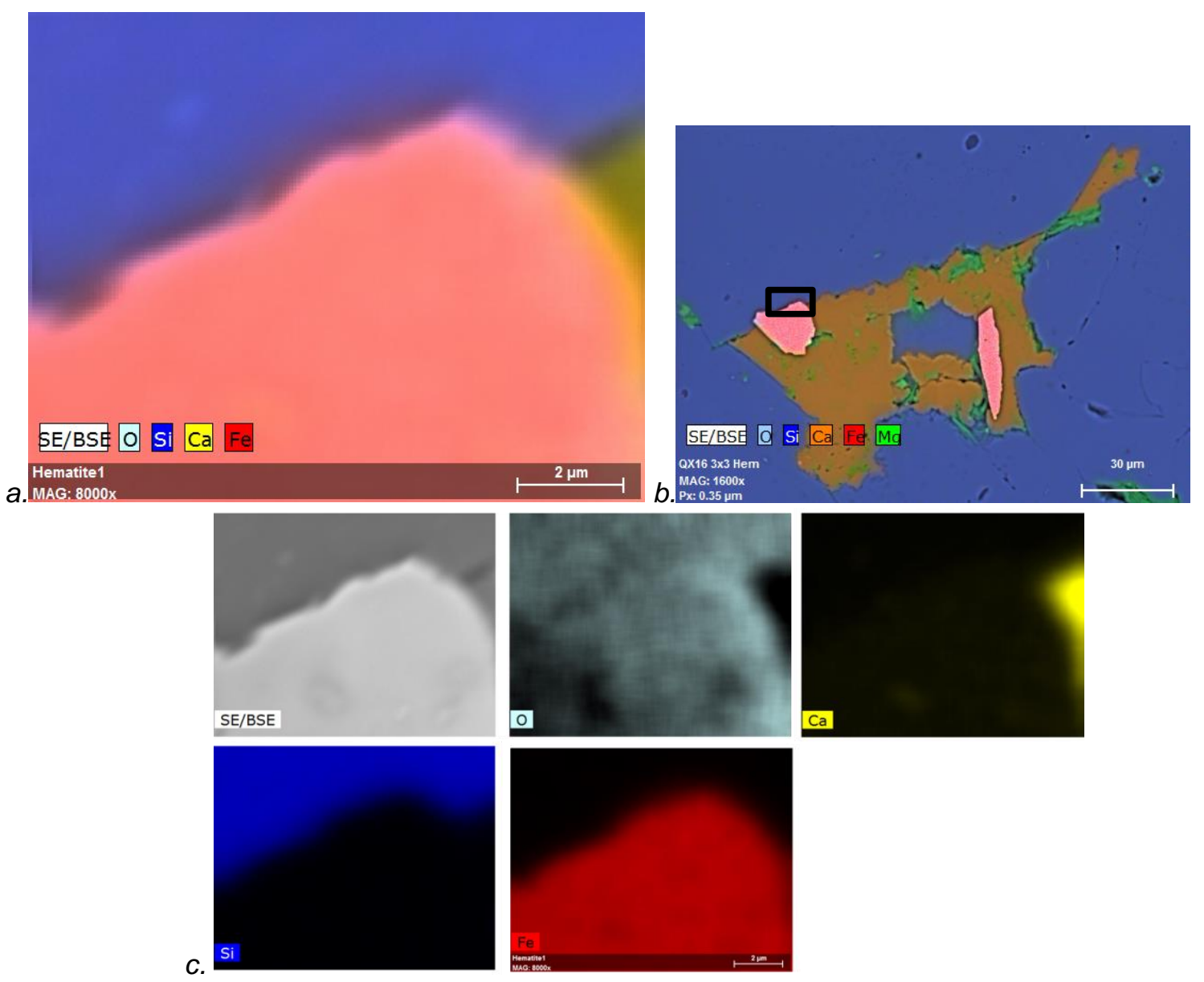

Fig. 9 SEM-EDX analysis of a portion of the hematite inclusion of Fig. 8 and its interface with quartz (top) and calcite (left) gangue matrix. The exact location across the large sample area is highlighted by the black square in Fig. 8b. a. collects the contribution from the different chemical elements, detailed in $\mathrm{c}$.

A smaller area of the top-left hematite inclusion was investigated using confocal micro-Raman spectroscopy, in order to univocally determine the kind of iron bearing oxide, since hematite, magnetite and goethite have distinct Raman spectra where it is more complicated to distinguish them with the SEM-EDX analysis only.

Single-point spectra were acquired on each scanning point of the target area. The resulting map is reported in Fig. 10. 
Additionally, spectra obtained by analysing a sample of pure hematite mineral, in powder form, are recorded. Single point spectra are reported in Fig. 11.

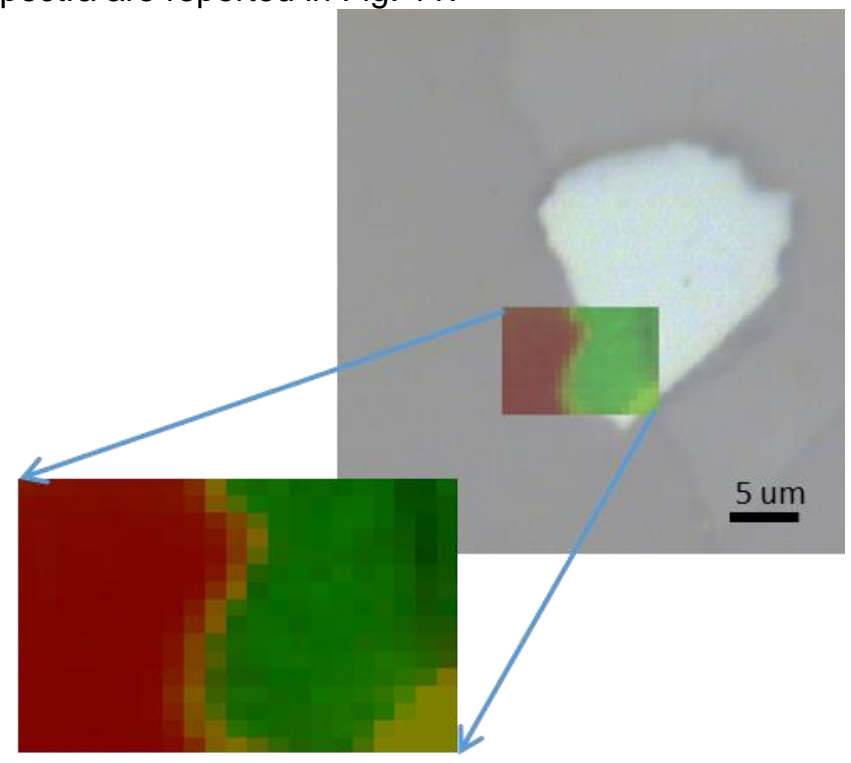

Fig. 10 Confocal micro Raman map (coloured) of the hematite inclusion overlapped with the optical image of the inclusion (in grey colour). The intensity of the $410.5 \mathrm{~cm}^{-1}$ band (green tone area) is a characteristic emission peak of the hematite, the intensity of the $463.0 \mathrm{~cm}^{-1}$ band (red tone area) is assigned to the quartz one.
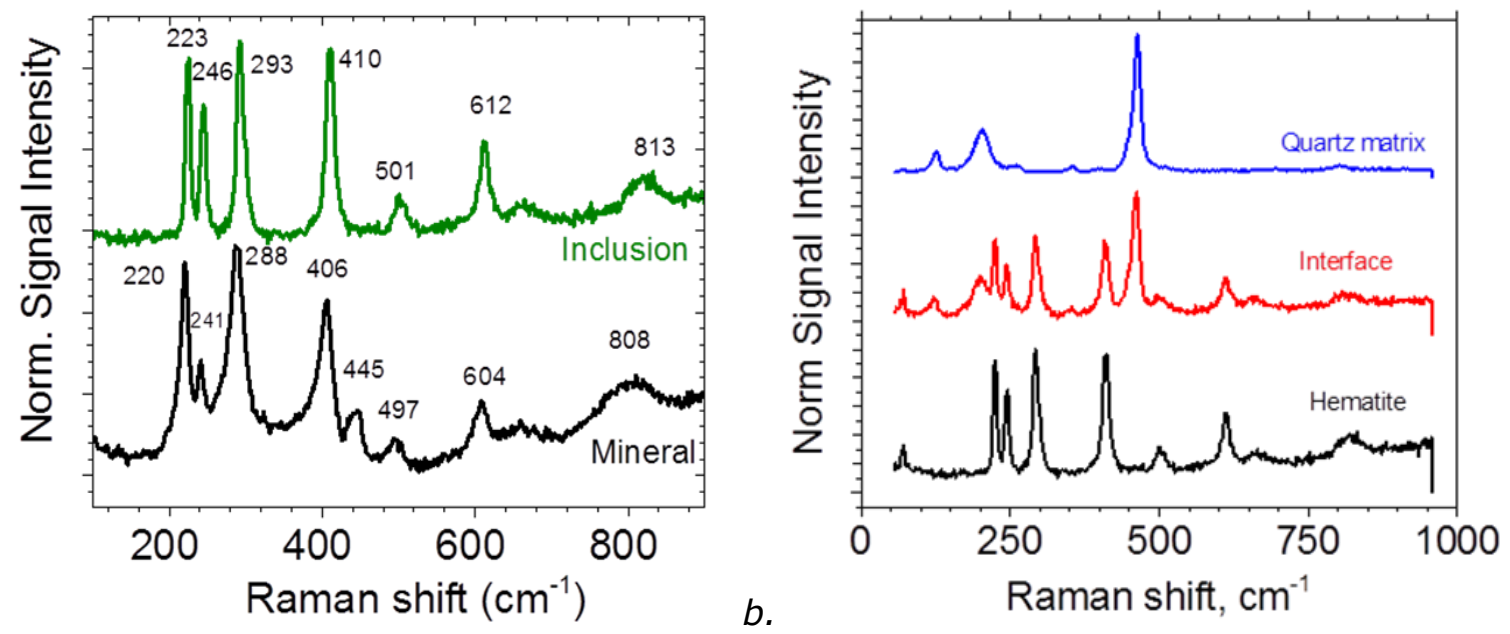

Fig. 11 a. Raman spectra of hematite inclusion (green) and powder of pure minerals analysed by the same system (black), which are the sum-average of seven spectra; b. Raman spectra of quartz, quartz-hematite interface and hematite suggesting that the interface is a mixture of two pure minerals (quartz and hematite).

\subsection{Scanning Microwave Microscopy results}

The SMM technique, described in previous sections, was applied to the hematite minerals, after their preliminary chemical and elemental characterization.

In order to quantify their dielectric constant, a calibration procedure (as described in Section 2.3) was applied before and after the measurements. Five materials with known dielectric constant (namely fused quartz $\mathrm{SiO}_{2}$, (100) magnesium oxide $\mathrm{MgO}$, (100) lanthanum aluminate $\mathrm{LaAlO}_{3}$, (110) titanium dioxide $\mathrm{TiO}_{2}$, (100) strontium titanate from CrysTec, $\mathrm{GmbH}$, Germany) were measured and the results were interpolated in the calibration curve in Fig. 5.

The microscopic image of the dielectric constant (namely SMIM-C as described in Section 2.3) of one of the hematite inclusions is shown in Fig. 12. An evident difference can be visually appreciated 
between the hematite (yellow) and the calcite (purple). The location and shape of the different elements from the SEM-EDX scan (Fig. 7) are clearly visible in the dielectric contrast image shown in Figure 12. An elongated small defect is also present in this left part of this image, at the hematitecalcite interface. The darker purple colour shows that the dielectric constant of such object is slightly lower than the calcite.
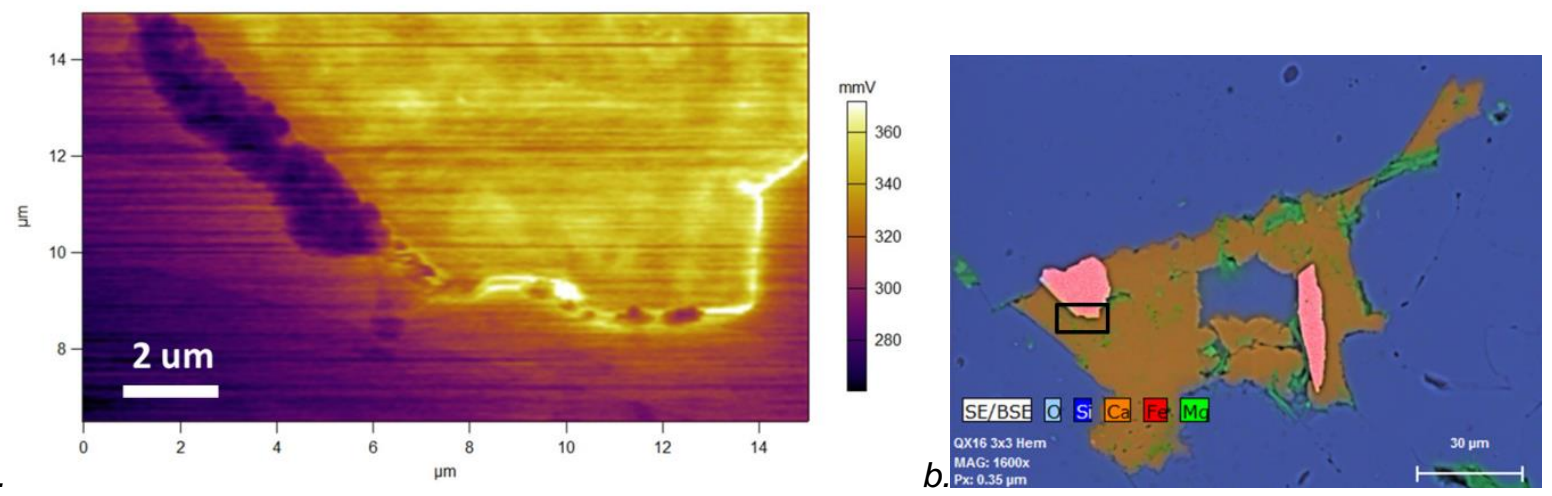

Fig. 12 a. Map of the $\Delta C$ data (sMIM-C) of the area under analysis. The dielectric contrast between hematite (yellow) and calcite (purple) is clearly visible. This picture highlights the dielectric contrast between the materials. $b$. On the SEM-EDX image of the entire sample area under investigation, the black square indicates the area of the sample analysed by the SMM in a.

It is then possible to convert the raw data, coming from the SMIM-C channel of the microscope, to real values of the dielectric constant through the calibration measurements performed before and after each experiment. Then a nanometric scale, quantitative map of such values is available for analysis (Fig. 13). Calcite and hematite are respectively identified by different colour tones (green/cyan, orange/yellow) that correspond to different values of $\varepsilon^{\prime}$.

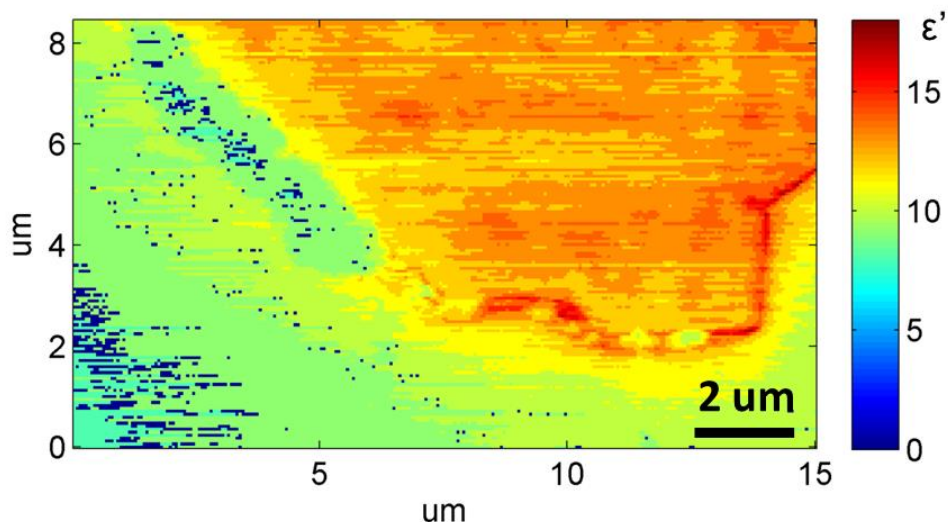

Fig. 13 Map of the dielectric constant $\varepsilon^{\prime}$ of the area under analysis. Calcite at bottom and left part of the image is green-cyan $\left(\varepsilon^{\prime}=[8-9]\right)$, central hematite is orange-yellow $\left(\varepsilon^{\prime}=[12-14]\right)$. The elongated horizontal artefacts are due to the occasional imperfect contact between tip and sample during the scanning. They are also visible in Fig. 12.

Finally, the loss tangent and conductivity of the hematite were evaluated. The proportionality constant $\kappa$ described above was obtained by the numerical model implemented in COMSOL Multiphysics ${ }^{\odot}$ and discussed in Section 3. From the experimental evaluation of the dielectric constant, the range $\varepsilon^{\prime}=[12-14]$ was assumed for the hematite inclusion and, from these assumptions, $\kappa=[8-9]$ was derived. Losses of the calcite (or quartz) matrix are negligible in the sMIM measurements and, therefore, not considered in this exemplifying analysis of the hematite minerals only. For the loss tangent of the hematite inclusion, shown in Fig. 13, values of $\varepsilon^{\prime \prime}=[8-15]$ and $\sigma=[1.5-2.6] \mathrm{S} / \mathrm{m}$ were obtained. We note that, in literature, the conductivity of the hematite was estimated to be within a range $\sigma=[0.1-1] \mathrm{S} / \mathrm{m}$ (Nelson et al., 1989; Peng et al., 2012; Hotta et al., 2010). 
Corresponding maps of the imaginary part of the complex dielectric permittivity $\left(\varepsilon^{\prime \prime}\right)$, of $\tan \delta$ obtained from equation (3), and of the microwave conductivity $\sigma$ of the inclusion are reported in Fig. 14. The surrounding gangue material has significantly lower electromagnetic losses at microwave frequencies than the hematite inclusion.

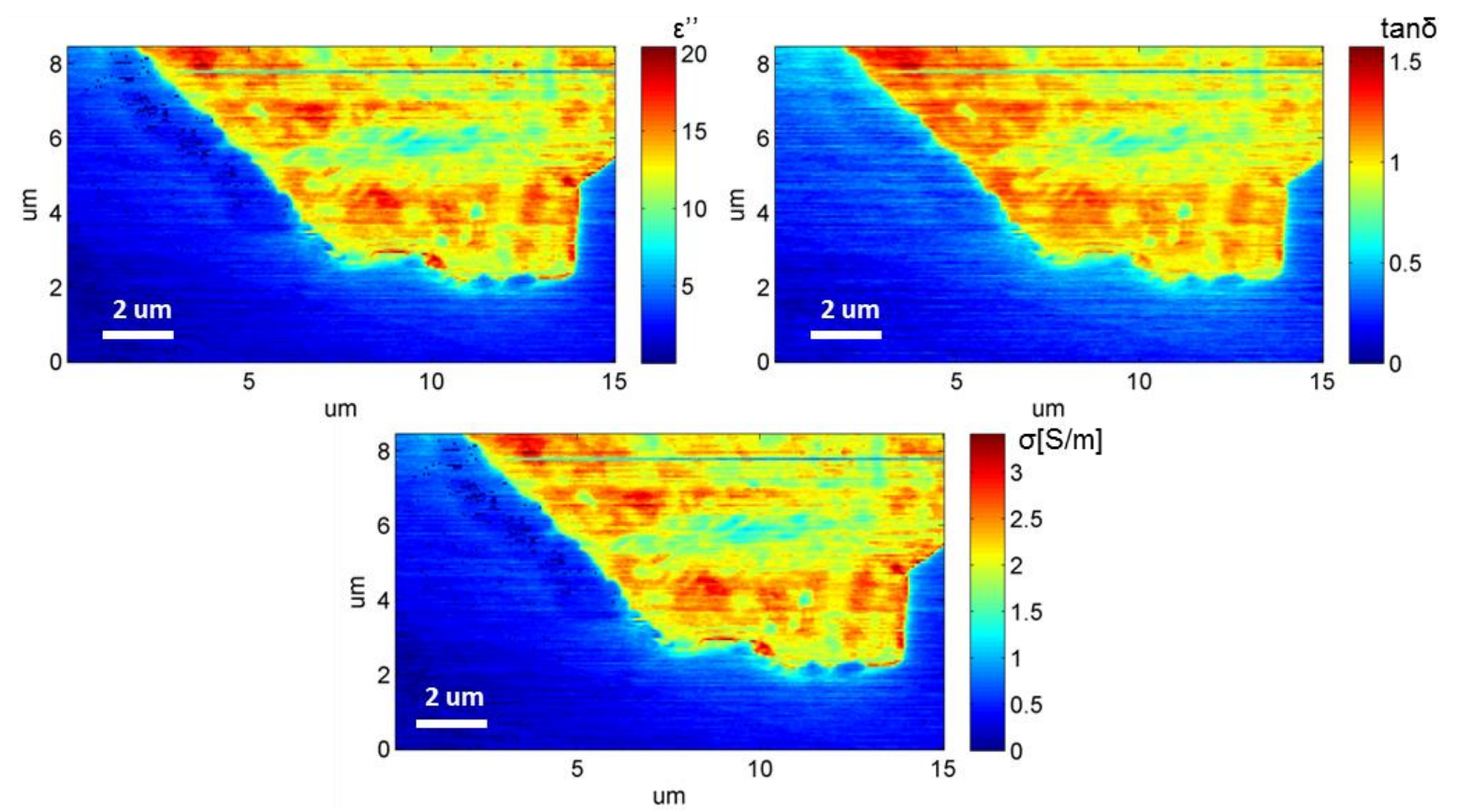

Fig. 14 Maps of the $\varepsilon "$, tan $\delta$ and $\sigma$ of the area under test. Note a certain heterogeneity in the maps of losses and conductivity within the hematite inclusion itself.

In order to verify the reproducibility of the technique, another inclusion was analysed by the SMM as well. Results are provided in Figs. 15 and 16.
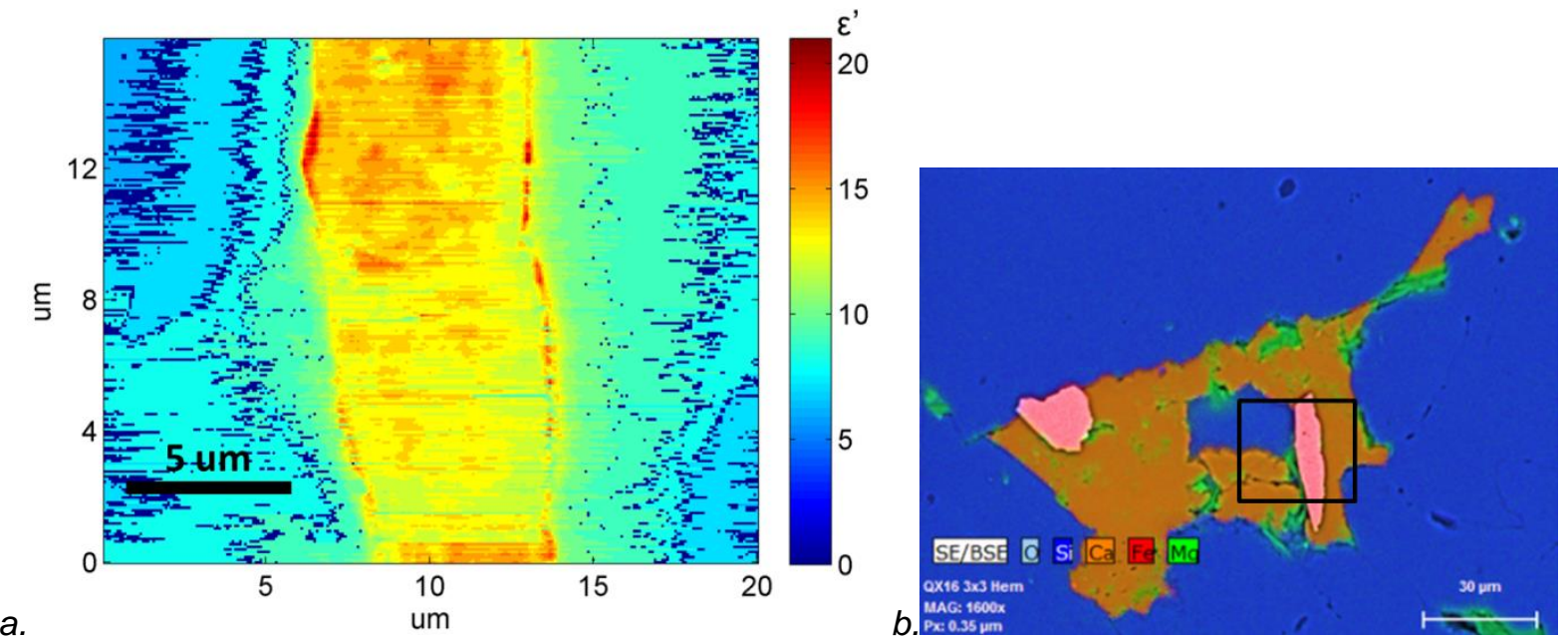

Fig. 15 a. Map of the dielectric constant $\varepsilon^{\prime}$ of the area under analysis (highlighted by the black box in b.). Quartz in the top-left corner and bottom-right one is blue $\left(\varepsilon^{\prime}=[4.5-5.5]\right)$, calcite around the elongated phase is cyan $\left(\varepsilon^{\prime}=[8-9]\right)$, central hematite is orange-yellow $\left(\varepsilon^{\prime}=[12-15]\right)$. As in Fig. 13 , the elongated artefacts are due to the occasional imperfect contact between tip and sample during the scanning. 


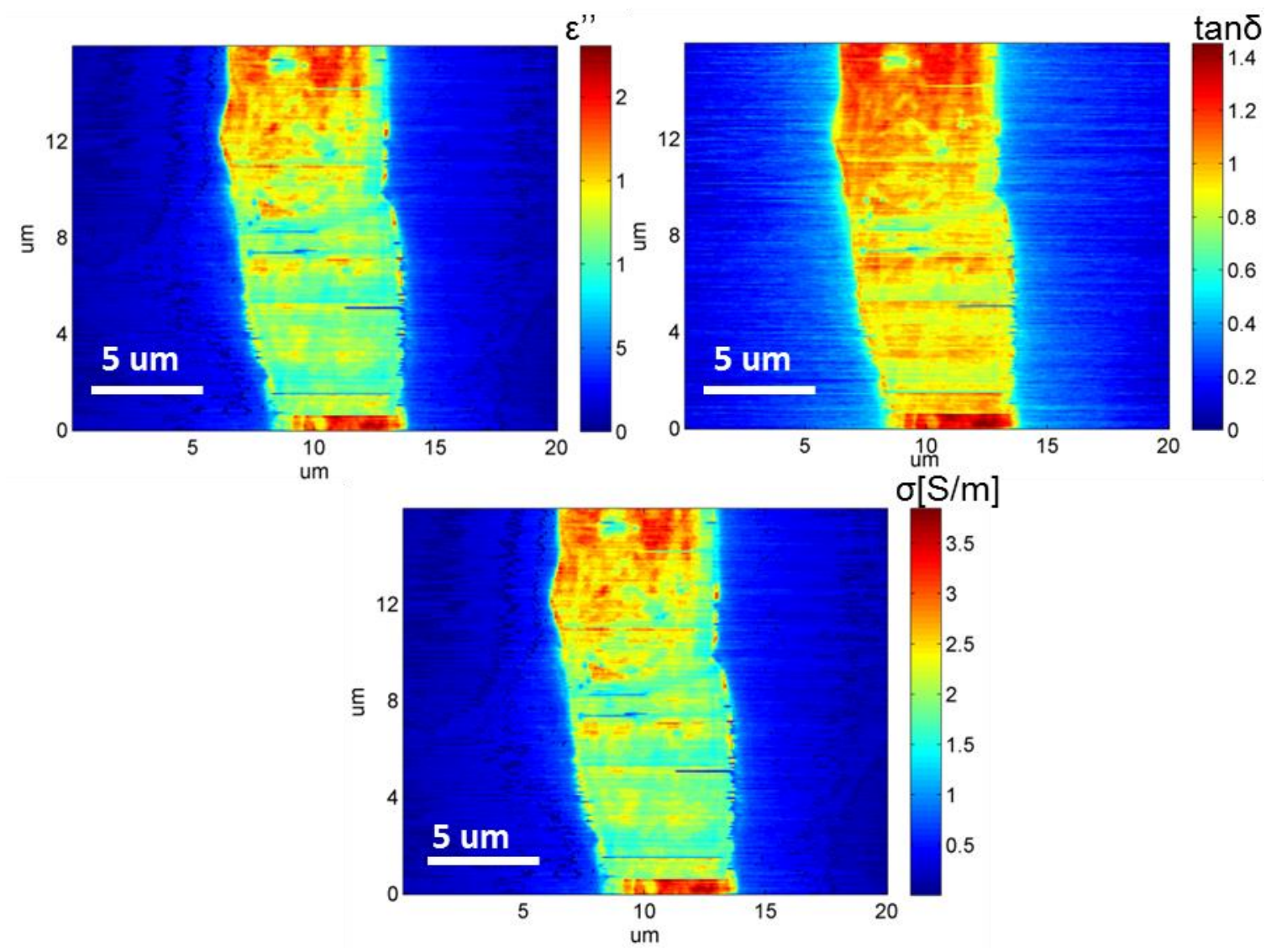

Fig. 16 Maps of the $\varepsilon "$, tan $\delta$ and $\sigma$ of the area under test. The difference between quartz and calcite is less evident than in Fig. 15. Note the heterogeneous map of conductivity within the hematite inclusion itself, with a range of variability comparable to the previous hematite inclusion.

Finally, in order to additionally verify the reproducibility of the method during time and the invariance to other parameters (such as scanned area of analysis or a different SMM tip) the first inclusion was tested with a blunter tip. The results of these experiments are reported in Figs. 18 and 19. In this case the sample was placed upside down with respect to previous analyses, hence the images are mirrored.

Calibration curve for this second tip is reported in Fig. 17. In this case the calibration was performed with 4 known loads (namely fused quartz $\mathrm{SiO}_{2}$, (100) magnesium oxide $\mathrm{MgO}$, (100) lanthanum aluminate $\mathrm{LaAlO}_{3}$, (110) titanium dioxide $\mathrm{TiO}_{2}$ from CrysTec, $\mathrm{GmbH}$, Germany), only up to $\varepsilon^{\prime}=100$.

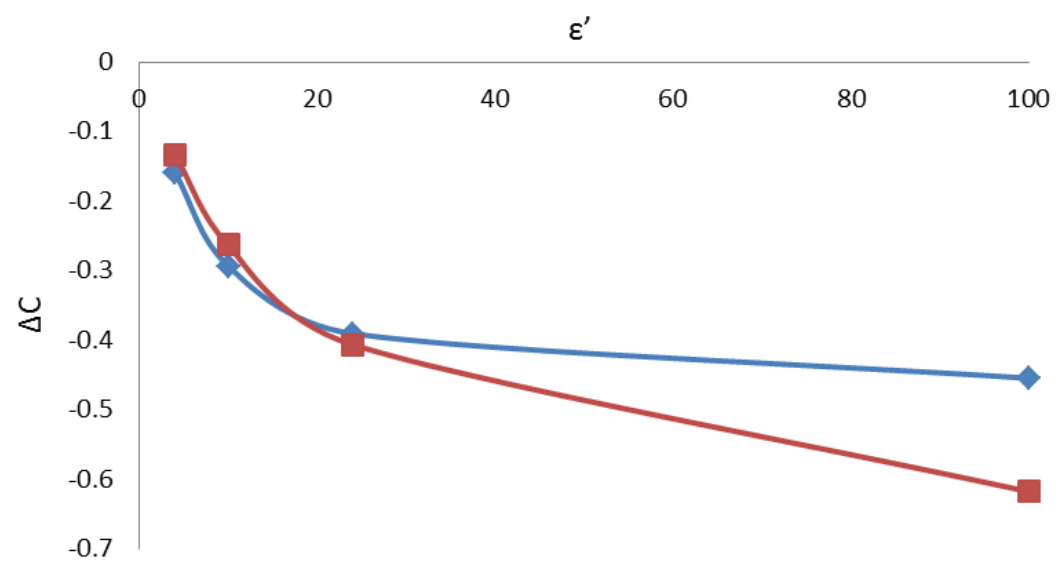

$\leadsto$ Pre-meas calibration $\quad-$ Post-meas calibration

Fig. 17 Calibration curve for $\varepsilon^{\prime}$ data, interpolating the values recorded by the C-channel of the microscope referred to the 'in air' condition $(\Delta C)$ on known dielectric samples $\left(\mathrm{SiO}_{2}\left(\varepsilon^{\prime} \approx 4\right), \mathrm{MgO}\right.$ 
$\left.\left(\varepsilon^{\prime} \approx 10\right), \mathrm{LaAlO}_{3}\left(\varepsilon^{\prime} \approx 24\right), \mathrm{TiO}_{2}\left(\varepsilon^{\prime} \approx 100\right)\right)$. The blue curve was measured before the rock analysis, the red one was obtained afterwards. The discrepancy between the two curves reflects the changes in size and shape of the SMM probe due to the contact with the sample during scanning.
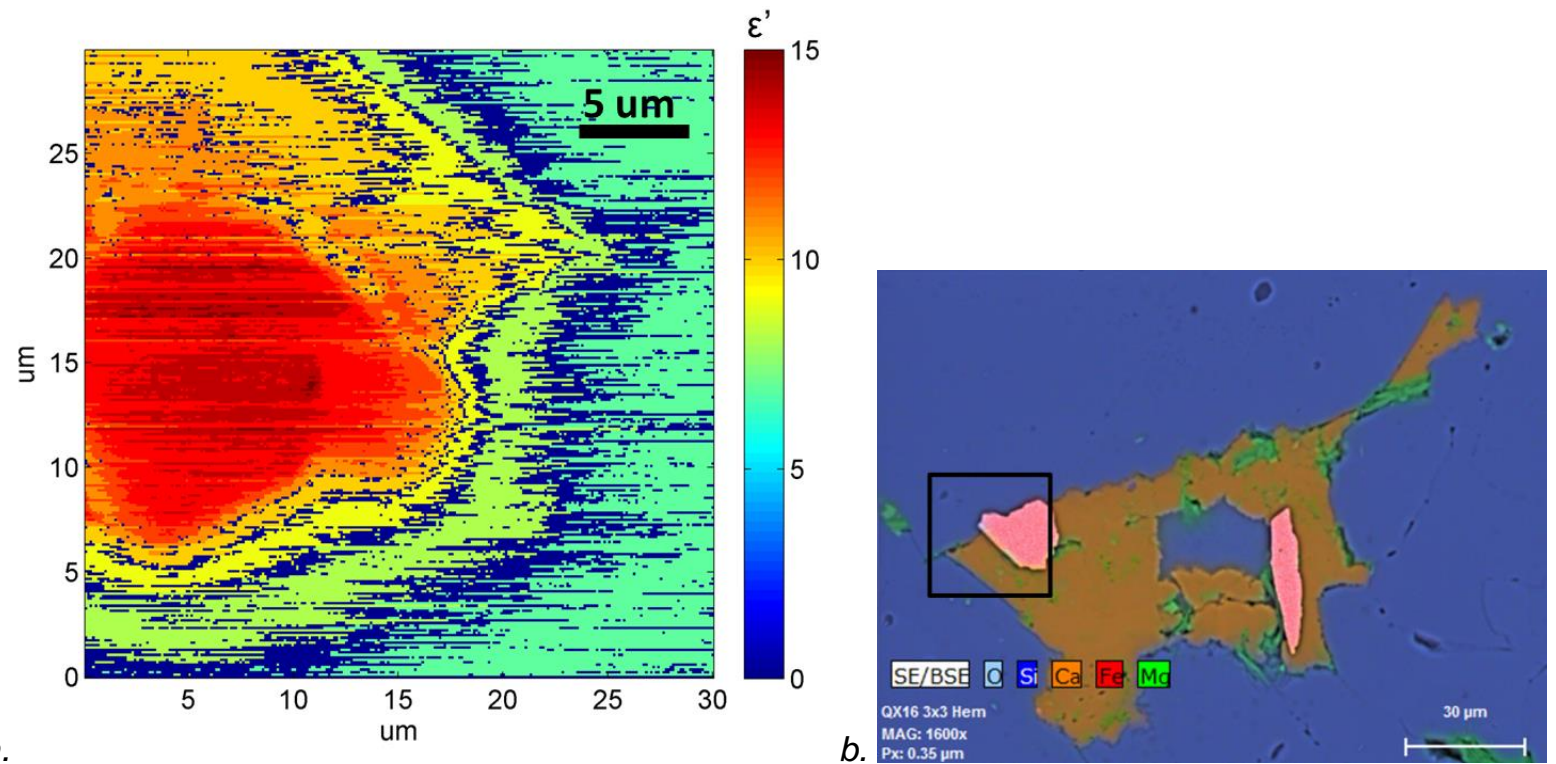

Fig. 18 a. Map of the dielectric constant $\varepsilon^{\prime}$ of the area under analysis (highlighted by the black box in b.). Quartz in the bottom and right parts of the image is blue/green $\left(\varepsilon^{\prime}=[5.5-6]\right)$, calcite at the top is yellow/orange $\left(\varepsilon^{\prime}=[9-10]\right)$, central-left hematite is red/dark red $\left(\varepsilon^{\prime}=[12-15]\right)$. As in Fig. 13, the elongated artefacts are due to the occasionally imperfect contact between tip and sample during scanning. 

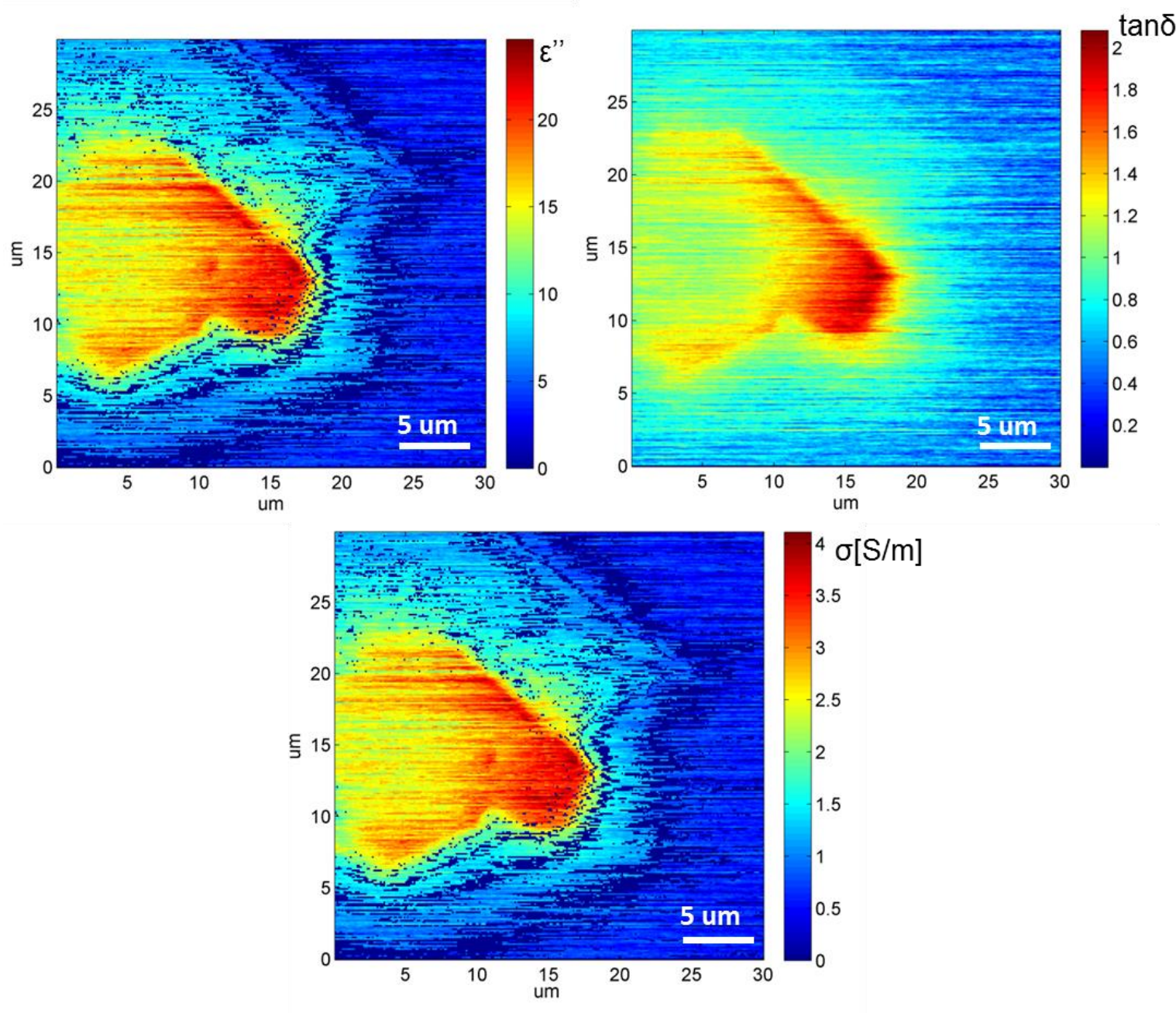

Fig. 19 Maps of the $\varepsilon^{\prime \prime}, \tan \delta$ and $\sigma$ of the area under test. The difference between quartz and calcite is less evident than in Fig. 18. Note the heterogeneous map of conductivity within the hematite inclusion itself, with a range of variability comparable to the previous hematite inclusion.

\section{Discussion}

\subsection{SEM-EDX and micro-Raman data discussion}

From the SEM-EDX analyses (see Fig. 7) it is possible to define the exact elemental composition of the mineral inclusions and the surrounding matrix. The images clearly highlight the presence of two spots whose composition is essentially Iron Oxide $(\mathrm{Fe}+\mathrm{O})$. Their size is less than 30 um.

They are embedded in a complex structure mainly identified as Calcite $(\mathrm{Ca}+\mathrm{C})$ with some $\mathrm{Mg}$ and $\mathrm{Al}$ contamination. Everything is surrounded by a wider quartz matrix $(\mathrm{Si}+\mathrm{O})$.

In order to compare the SEM-EDX mineral map images with the micro-Raman maps and with the SMM ones, SEM-EDX analyses were performed on smaller areas; results are reported in Figs. 8, and 9. In Fig. 8, the minerals present in the region mapped include $\mathrm{Fe}, \mathrm{Si} \mathrm{Ca} \mathrm{Fe}, \mathrm{O}$ and a few $\mathrm{Mg}$ rich minerals. A combined colour image of all the 6 identified minerals present in the region is presented in Figs. 8a. and 9a. Similar results are reported for the smaller area analysed in Fig. 9. The resolution is poorer since it is reaching the limit of the SEM-EDX system itself.

In Fig. 10 an exemplar micro-Raman map is reported. Three different areas are distinguishable by overlapping two colour maps related to $410.5 \mathrm{~cm}^{-1}$ and to $463.0 \mathrm{~cm}^{-1}$. The first one (green tones) is related to one of the typical emission peaks of the hematite; the second one (red tones) is related to a typical emission peak of the quartz. In the right-bottom corner it is possible to see the contribution from the calcite interface. 
In order to depict a complete analysis of the complex rock structure, calcite should also be included. One of the limitations of Raman confocal mapping is that it is optimized for a scan at constant power of the laser excitation and highest signal to noise ratio for all constituents. In the case of calcite, we found it exhibited significant fluorescence, which complicated the experiment and subsequent spectral analysis. The origins of calcite fluorescence are well known (http://www.fluomin.org/uk/fiche.php?id=157) and related to substitutional doping (for instance with $\mathrm{Mn}^{+2}$ or $\mathrm{Pb}^{+2}$ or $\mathrm{Ce}^{+3}$ among many). For this reason it is not possible to add a meaningful contribution from the calcite to the map and then in this work the Raman measurements exclude the calcite characterization.

The hematite area under test has a rich Raman spectrum with the peaks at 223, 246, 293, 410 and $612 \mathrm{~cm}^{-1}$. Subsequently, a Raman spectrum obtained by sum-averaging spectra at 7 positions on an inclusion of the iron oxide, was compared to the spectra obtained by analysing a sample of pure hematite mineral. Results are reported in Fig. 11a.

Those results were previously reported in the literature (https://www.fis.unipr.it/phevix/ramandb.php?plot=Hematite1\&submit=Go).

The Raman spectrum of $\alpha-\mathrm{Fe}_{2} \mathrm{O}_{3}$, hematite, exhibits seven peaks which were previously assigned to $A_{1 g}$ mode (225 and $498 \mathrm{~cm}^{-1}$ ) and five peaks of $E_{g}$ modes (247, 293, 299, 412 and $613 \mathrm{~cm}^{-1}$ peaks) (Porto and Krishnan, 1967; Beattie and Gibson, 1970).

In both the inclusion and the pure hematite sample, a broad band at about $1300 \mathrm{~cm}^{-1}$ was observed (not shown on Fig. 11a.) which was previously assigned to the collective spin movement (two-magnon scattering) which is very sensitive to temperature (Thibeau, 1978). The condition of the Raman acquisition was maintained such as to minimize any heating effect, which is known to shift peak positions as well as to cause peak broadening.

Slight differences in the Raman spectrum of the inclusion compared to the pure hematite spectrum, especially in the relative intensity of the two major $A_{1 g}$ and $E_{g}$ bands at 225 and $293 \mathrm{~cm}^{-1}$ stems from the unknown orientation of the inclusion crystal lattice in respect to the laser polarization. Also it allows the identification of the inclusion as high purity hematite.

The Raman spectra of quartz, quartz-hematite interface and hematite in Fig. 11b. suggest that the interface is a mixture of two pure minerals (quartz and hematite), while the minerals appears uniform to the Raman analysis. The quartz spectrum suggests that $\mathrm{SiO}_{2}$ is present in the $\alpha$-quartz polymorph, based on previous assignment by Kingma and Hemley (1994).

\subsection{Scanning Microwave Microscopy data discussion}

The SMM analysis in this work was focused on the two hematite inclusions depicted in Fig. 7. Reproducibility of the method was demonstrated by analysing both the inclusions and by employing two different SMM probes. Calibrated data of the dielectric constant of Figs. 13, 15 and 18 shows good reproducibility among the inclusions and with different tip conditions. Additionally, the results are independent from the size of the scanned area and from the size of the inclusions.

These data are in good agreement with earlier literature results, taking into account a wide variability of conditions and differences between the measurement methods. For example, in Nelson, et al. (1989), the dielectric values for the bulk density of the material were derived from theoretical calculations, while the measurements were performed on powders of pure minerals at different densities. In Peng et al. (2012) the complex dielectric permittivity data was measured by cavity perturbation technique using hematite samples composed of powders of pure minerals with a bulk density approaching that of the solid. It is not possible to directly compare Peng et al. results with this study: the measurements that they performed with a powder density closer to that of a solid material were influenced by thermal effects. In Hotta, et al. (2010), the open-ended coaxial probe technique was used on compressed powders of pure hematite crystals with different densities. As expected, the trend of the $\varepsilon$ ' and $\varepsilon$ " with the relative density was increasing; the exact values were still obtained by numerical calculations. The results reported in this work are in overall agreement with the estimation given by Hotta, et al. for the case of relative density unitary. 
In this work, the SMM data are referred to the real and local density of the sample under test. There is no need for complex dielectric mixture equations to determine the dielectric properties of the sample in its natural condition. Furthermore, the SMM probe can locally test the effects of defects in the inclusions under test. This is the case for the hematite inclusion in Fig. 15. The corresponding AFM image is reported in Fig. 20. Two pinch defects (highlighted by the red circles) are vaguely visible in the map of Fig. 15a., indicating that there is only a small topographical cross-talk in the sMIM-C signal, but it is still possible to identify the spots in the loss maps of Fig. 16.

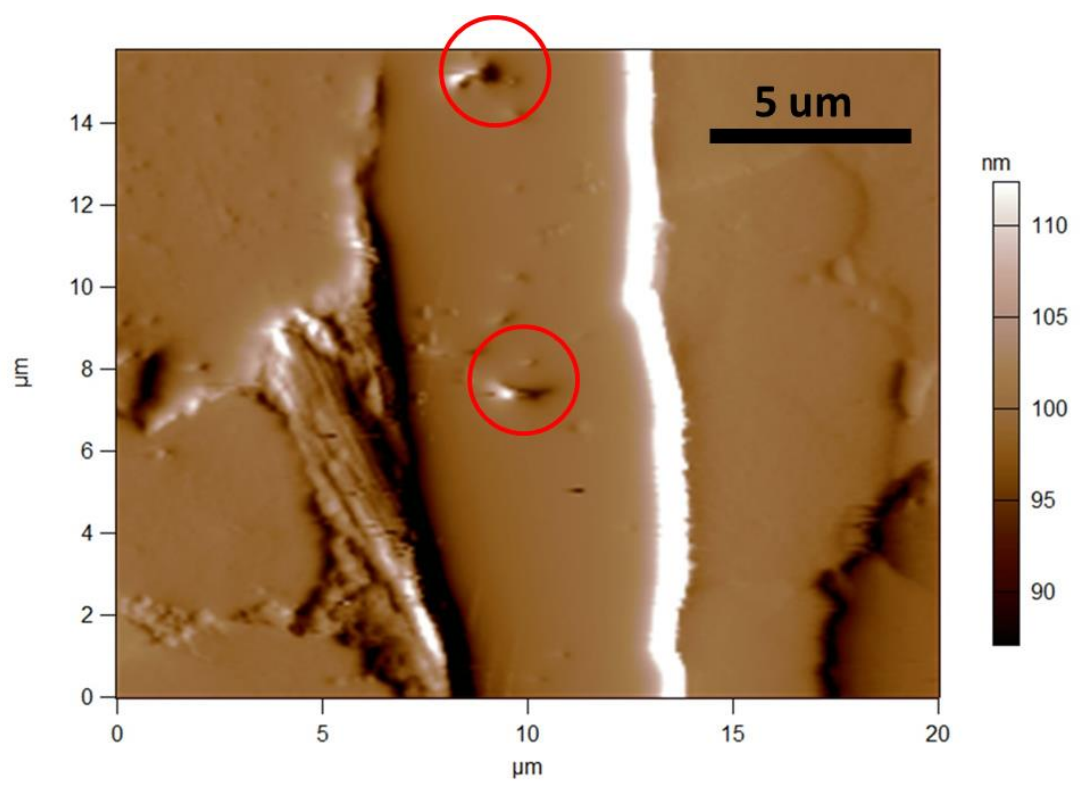

Fig. 20 AFM topography of the same area under test reported in Fig. 15a. The scanned surface is flat within few $\mathrm{nm}$. The hematite surface is flat with few pinch defects on its surface, still recognizable in the related dielectric maps.

Another interesting feature is a variability, which is especially evident in the loss images, along the entire surface of the inclusions. Several micrometric areas in the hematite in Figs. 14 and 16 show a higher conductivity than the rest of the phase. However, such variability was not found in SEM-EDX and micro-Raman analysis. Additionally, the AFM topography does not show a similar pattern, and only pinch defects are seen on the hematite surface (Figs. 20,21).
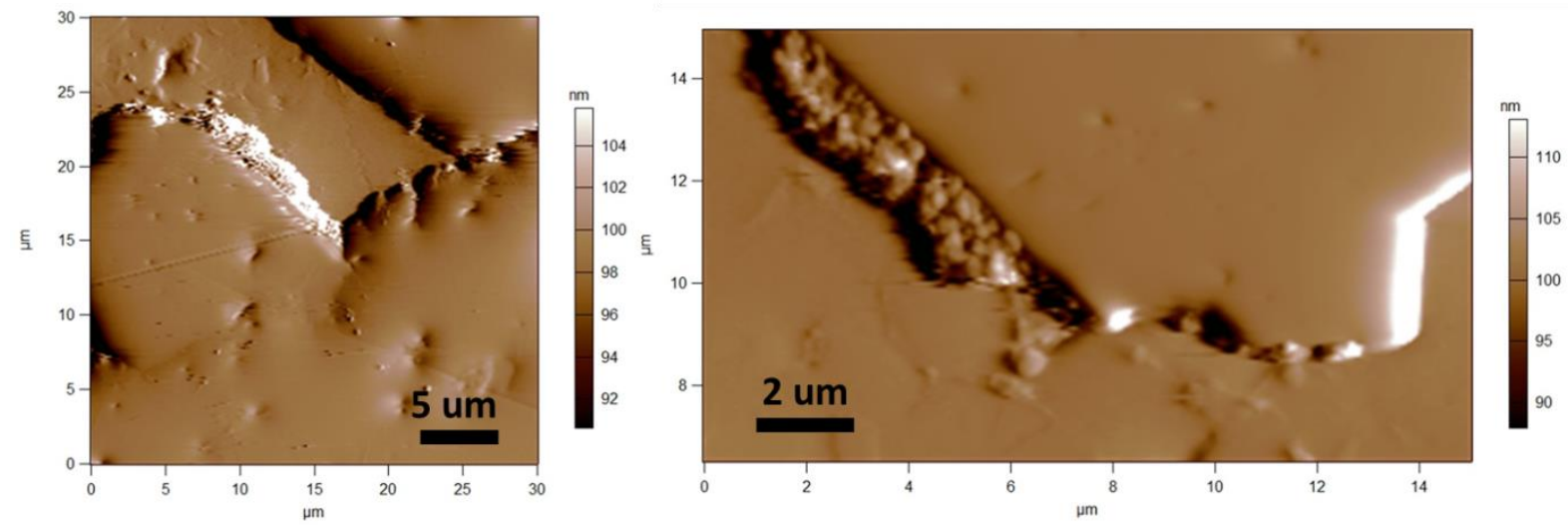

Fig. 21 AFM topography of the same areas under test reported in Figs. 18a and 12a, respectively. The scanned surface is flat within few $\mathrm{nm}$. The hematite surface is flat with few pinch defects on its surface, still recognizable in the related dielectric maps.

Such variability at micrometric scale is richly detailed in the results of the analysis with the sharper SMM tip (Figs 14, 16) and less detailed in Fig. 19. In the latter case, the probing was performed with a blunter tip, and therefore, it is likely that smaller details are averaged with the surrounding and unresolved in the images. 
The limited variation recorded in the conductivity can be ascribed to intrinsic variability of the hematite itself (localized differences in density, orientation of the crystal structure) that cannot be recorded by either SEM-EDX or micro-Raman technique.

The unique working scale of the scanning microwave microscope could be efficiently used for quantifying the dielectric properties of inclusions within mineral samples and also for relating such properties to their physical structure, phases and chemical composition at a similar scale. A similar study, where dielectric properties were related to material composition, was performed on homogeneous samples by measuring the dielectric properties with conventional 'bulk' techniques (Yoon et al., 2006).

\section{Conclusions}

In this work we demonstrated nanoscale dielectric characterization of mineral inclusions within a natural rock with a complex composition. In particular, two micrometric-sized inclusions of hematite, embedded in a gangue matrix, were analysed by a scanning microwave microscope, and details about its dielectric constant, losses and conductivity were presented. All data are in good agreement with previous literature for pure mineral samples. The dielectric constant of the hematite inclusion was found in the range $\varepsilon^{\prime}=[12-15]$ while the conductivity was estimated to be between $\sigma=$ $[1.2-2.5] \mathrm{S} / \mathrm{m}$, with a limited variability within each inclusion. Dielectric properties of calcite and quartz were mapped as well; their values are in the ranges $\varepsilon^{\prime}=[8-10]$ and $\varepsilon^{\prime}=[4.5-6]$, respectively. The results from the scanning electron microscopy/energy-dispersive x-ray spectroscopy analysis and micro Raman analysis are in agreement with the dielectric maps obtained by the scanning microwave microscopy. The hematite grains were found to be chemically pure, and the corresponding dielectric maps are essentially uniform. The capability of the scanning microwave microscope for measurement of the dielectric properties of a mineral inclusion at a micrometric length scale was demonstrated. The limited variation recorded in the conductivity can be ascribed to intrinsic variability of the hematite itself (localized differences in density, orientation of the crystal structure) that cannot be detected by either SEM-EDX or micro-Raman technique.

The unique capability of the scanning microwave microscope can be efficiently used for quantification of the dielectric properties of inclusions within mineral samples at the micrometre length scale and below as well as for relating such properties to their physical structure, phases and chemical composition at a similar scale. Additionally, the SMM data can be potentially used in multi-physics studies of the complex interactions between microwaves and rocks in order to accurately model the complex heterogeneity of the rocks in terms of dielectric properties.

\section{Acknowledgement}

Microwave microscopy and confocal micro-Raman spectroscopy measurements were performed at Center for Nanophase Materials Sciences, which is a Department of Energy Office of Science User Facility.

\section{References}

Ali, A. Y., Bradshaw, S. M., 2009. Quantifying damage around grain boundaries in microwave treated ores. Chemical Engineering and Processing: Process Intensification 48 (11-12), 1566-1573.

Ali, A. Y., Bradshaw, S. M., 2010. Bonded-particle modelling of microwave-induced damage in ore particles. Minerals Engineering 23 (10), 780-790.

Ali, A. Y., Bradshaw, S. M., 2011. Confined particle bed breakage of microwave treated and untreated ores. Minerals Engineering 24 (14), 1625-1630.

Anlage, S. M., Talanov, V. V., Schwartz, A. R., 2007. Principles of near-field microwave microscopy, in: Kalinin, S., Gruverman, A., Scanning probe microscopy: electrical and electromechanical phenomena at the nanoscale. Springer Scientific: New York, 215-253.

Binnig, G., Rohrer, H., 1983. Scanning tunneling microscopy. Surface Science 126 (1), 236-244. 
Binnig, G., Quate, C. F., Gerber, Ch., 1986. Atomic force microscope. Physical Review Letters 56 (9), 930-934.

Beattie, I. R., Gilson, T. R., 1970. The single-crystal Raman spectra of nearly opaque materials. Iron(III) oxide and chromium(III) oxide. Journal of Chemical Society A, 980-986.

Collins, L., Tselev, A., Jesse, S., Okatan, M. B., Proksch, R., Mathews, J. P., Mitchell, G. D., Rodriguez, B. J., Kalinin, S. V., Ivanov, I. N., 2014. Breaking the limits of structural and mechanical imaging of the heterogeneous structure of coal macerals. Nanotechnology 25 (43), 435402 (9pp).

Colthup, N. B., Daly, L. H., Wiberley, S. E., 1975. Introduction to infrared and Raman spectroscopy. $2^{\text {nd }}$ edition, Academic Press, Inc. (London) Itd.

Database of luminescent Minerals http://www.fluomin.org/uk/fiche.php?id=157, last access 15/04/2016.

Haque, K. E., 1999. Microwave energy for mineral treatment processes-a brief review. International Journal of Mineral Processing 57 (1), 1-24.

Hartlieb, P., Leindl, M., Kuchar, F., Antretter, T., Moser, P., 2012. Damage of basalt induced by microwave irradiation. Minerals Engineering 31, 82-89.

Hartlieb, P., Toifl, M., Kuchar, F., Meisels, R., Antretter, T., 2015. Thermo-physical properties of selected hard rocks and their relation to microwave-assisted comminution. Minerals Engineering, in press.

Hotta, M., Hayashi, M., Nagata, K., 2010. Complex permittivity and permeability of $\alpha-\mathrm{Fe} 2 \mathrm{O} 3$ and Fe1-xO powders in the microwave frequency range between 0.2 and $13.5 \mathrm{GHz}$. ISIJ International 50 (10), 1514-1516.

John, R. S., Batchelor, A. R., Ivanov, D., Udoudo, O. B., Jones, A. D., Dodds, C., Kingman, S. W., 2015. Understanding microwave induced sorting of porphyry copper ores. Minerals Engineering 84, 77-87.

Jones, D. A., Kingman, S. W., Whittles, D. N., Lowndes, I. S., 2005. Understanding microwave assisted breakage. Minerals Engineering 18 (7), 659-669.

Kingma, K., Hemley, R. J., 1994. Raman spectroscopic study of microcrystalline silica. American Mineralogist 79, 269-273.

Kingman, S. W., Rowson, N. A., 1998. Microwave treatment of minerals-a review. Minerals Engineering 11 (11), 1081-1087.

Kingman, S. W., Vorster, W., Rowson N. A., 2000. The influence of mineralogy on microwave assisted grinding. Minerals Engineering 13 (3) 313-327.

Kingman, S. W., Jackson, K., Bradshaw, S. M., Rowson, N. A., Greenwood, R., 2004. An investigation into the influence of microwave treatment on mineral ore comminution. Powder Technology 146 (3), 176-184.

Kingman, S. W., 2006. Recent developments in microwave processing of minerals. International Materials Reviews 51 (1), 1-12.

Meisels, R., Toifl, M., Hartlieb, P., Kuchar, F., Antretter, T., 2015. Microwave propagation and absorption and its thermo-mechanical consequences in heterogeneous rocks. International Journal of Mineral Processing 135, 40-51.

Metaxas, A. C., Meredith, R. J., 1983. Industrial microwave heating, ch.4. IET Power and Energy series 4. The Institution of Engineering and Technology, London, United Kingdom. 
Nelson, S. O., Lindroth, D. P., Blake, R. L., 1989. Dielectric properties of selected minerals at 1 to 22 GHz. Geophysics 54 (10), 1344-1349.

Peng, Z., Hwang, J.-Y., Park, C.-L., Kim, B.-G., Andriese, M., Wang, X., 2012. Microwave permittivity, permeability, and absorption capability of ferric oxide. ISIJ International 52 (9), 1535-1538.

Pickles, C. A., Mouris, J., Hutcheon, R. M., 2005. High-temperature dielectric properties of goethite from 400 to $3000 \mathrm{MHz}$. Journal of Materials Research 20 (1), 18-29.

Porto, S. P. S., Krishnan, R. S., 1967. Raman effect of corundum. The journal of chemical physics 47 (3), 1009-1012.

Prime Nano Inc. website http://www.primenanoinc.com/, last access 15/04/2016.

Raman database of the Laboratory of Photoinduced Effects Vibrational and X-Ray Spectroscopies of the University of Parma https://www.fis.unipr.it/phevix/ramandb.php?plot=Hematite1\&submit=Go, last access 15/04/2016.

Roberts, S., Von Hippel, A., 1946. A new method for measuring dielectric constant and loss in the range of centimeter waves. Journal of Applied Physics 17, 610.

Salsman, J. B., Holderfield, S. P., A technique for measuring the dielectric properties of minerals at microwave heating frequencies using an open-ended coaxial line, US Department of Interior, Bureau of Mines, 1994.

Salsman, J. B., Williamson, R. L., Tolley, W. K., Rice, D. A., 1996. Short-pulse microwave treatment of disseminated sulfide ores. Minerals Engineering 9 (1), 43-54.

Shivola, A., 1999. Electromagnetic mixing formulas and applications. IET Electromagnetic Waves Series 47. The Institute of Engineering and Technology, London, United Kingdom.

Tinga, W. R., 1989. Microwave dielectric constants of metal oxides at high-temperature, part 1 and part 2. Electromagnetic Energy Reviews 2 (1), 349-351.

Thibeau, R. J., Brown, C. W., Heidersbach, R .H., 1978. Raman spectra of possible corrosion products of iron. Applied Spectroscopy 32 (6), 532-535.

Toifl, M., Meisels, R., Hartlieb, P., Kuchar, F., Antretter, T., 2016. 3D numerical study on microwave induced stresses in inhomogeneous hard rocks. Minerals Engineering, in press.

Walkiewicz, J. W., Kazonich, G., McGill, S. L., 1988. Microwave heating characteristics of selected minerals and compounds. Minerals and Metallurgical Processing 5 (1), 39-42.

Walkiewicz, J. W., Raddatz, A. E., McGill, S. L., 1989. Microwave-assisted grinding. Industry Applications Society Annual Meeting 2 (1-5), 1528-1532.

Whittles, D. N., Kingman, S., Lowndes, I., Jackson, K., 2006. Laboratory and numerical investigation into the characteristics of rock fragmentation. Minerals Engineering 19 (14), 1418-1429.

Yoon, S. H., Kim, D.-W., Cho, S.-Y., Hong, K. S., 2006. Investigation of the relations between structure and microwave dielectric properties of divalent metal tungstate compounds. Journal of the European Ceramic Society 26 (10-11), 2051-2054. 